\title{
Stability Analysis and Design of Nonlinear Sampled-Data Systems Under Aperiodic Samplings
}

\author{
K.Hooshmandi ${ }^{1}$, F. Bayat ${ }^{2 * \dagger}$, M. R. Jahed-Motlagh ${ }^{1}$, A. A. Jalali ${ }^{1}$ \\ ${ }^{1}$ Department of Electrical Engineering, Iran University of Science and Technology, Tehran, Iran. \\ ${ }^{2}$ Department of Engineering, University of Zanjan, Zanjan, Iran.
}

\begin{abstract}
SUMMARY
In this paper, the problem of exponential stability analysis and design of sampled-data nonlinear systems have been studied using a polytopic LPV approach. By means of modeling a new double-layer polytopic formulation for nonlinear sampled-data systems, a modified form of piecewise continuous LyapunovKrasovskii functional is proposed. This approach provides less conservative robust exponential stability conditions by using Wirtingers inequality in terms of linear matrix inequalities. The distances between the real continuous parameters of the plant and the measured parameters of the controller are modeled by convex sets, and the analysis/design conditions are given at the vertices of some hyper-rectangles. In order to get tractable LMI conditions for the stabilization problem, we performed relaxation by introducing a slack variable matrix. Under the new stability criteria, an approach is introduced to synthesize a sampleddata polytopic LPV controller considering some constraints on the location of the closed-loop poles in the presence of uncertainties on the varying parameters. It is shown that the proposed controller guarantees the exponential stability of the closed-loop system for aperiodic sampling periods smaller than a known value, i.e. maximum allowable sampling period (MASP). Finally, the effectiveness of the proposed approach is verified and compared with some state-of-the-art existing approaches through numerical simulations. Copyright (c) 2010 John Wiley \& Sons, Ltd.
\end{abstract}

KEY WORDS: Nonlinear sampled-data systems; LPV systems; Lyapunov-Krasovskii functional; Wirtingers inequalities; LMI method.

\section{INTRODUCTION}

In many practical applications, the use of communication channels to implement control algorithms has considerably increased. In such applications, a heavy temporary load of computation in a processor and communication constraints can corrupt the sampling period of a given controller. Therefore, the variation of the sampling period will affect the stability and the performance of the closed loop system. So, it is quite important to determine a maximum allowable delay that the closed loop system remains stable for a given controller [1, 2, 3, 4]. The sampled-data control systems are prevalent approaches for stability analysis and controller design for systems under aperiodic sampling. Different techniques, including lifting approach [5], robust approach [6], impulsive model

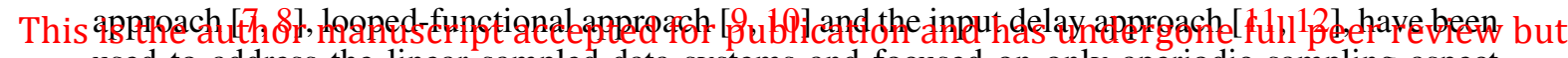
used to address the linear sampled-data systems and focused on only aperiodic sampling aspect has not. peen through the copyediting tytesetting, pagination and proofreading process, which may lead to differences between this version and the Version of Record. Please cite this article

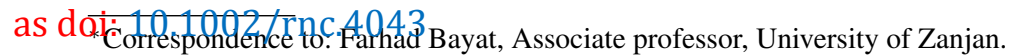

${ }^{\dagger}$ E-mail: bayat.farhad@znu.ac.ir 
Nonlinear sampled-data systems arise when either the plant model or the controller is nonlinear. While their linear counterpart is now a mature area, nonlinear sampled-data systems are much harder to deal with and, hence, much less studied. Therefore, there are a few references in the literature of nonlinear sampled-data systems where the input delay model [13, 14] or hybrid model [15] of the system is studied. Their inherent complexity leads to a closed-loop system which is mathematically involved $[13,14,15]$ and thus, can not be directly used to design a controller that provides a desired maximum allowable sampling periods (MASP). They only developed Lyapunovlike sufficient conditions for robust global asymptotic stability or input-to-output stability of a generally nonlinear sampled-data system. In contrast, $[16,17]$ provide linear state feedback sampled data controller design condition for nonlinear system with sector-bounded nonlinearity based on the input delay approach and using a different LKF. The polytopic LPV (PLPV) systems are suitable for the sampled-data control of nonlinear systems since many nonlinear systems can be modeled by a linear system with some time varying parameters $[18,19]$. As a consequence, some powerful design methods in the linear systems can be effectively extended to the PLPV systems, so that stability analysis and design condition with sampled-data PLPV state feedback controller lead to convex optimization problem in the framework of LMIs. Using PLPV model for stability analysis and design of nonlinear sampled-data systems faces the challenge of non-compliance between the actual and the measured parameters. This issue is addressed in this paper by analyzing the impact of the sampling interval and the rate of parameters variations on the stability and stabilization of the closed-loop sampled-data system where the system is described by a double-layer polytopic model.

In the context of LPV systems, there are few research papers formally addressing the analysis of the sampled-data problem. In [20,21], assuming that the parameters do not vary in the interval between consecutive sampling, a lifting technique is applied to derive stability conditions. A timedelay approach is utilised by $[22,23,24]$ for sampled-data controller design of continuous time LPV systems through mapping the hybrid closed-loop system to a continuous-time state-delayed LPV system. Stability conditions are derived using a smooth LKF which utilised in stability analysis of systems with fast varying input delays. Reference [22] considered the sampled parameter vector as a new parameter which make the optimization problem corresponding to the sampled-data controller design so conservative while in $[23,24]$ it is assumed that the time-varying parameters are constant between two consecutive samples which is restricted to slowly parameter varying systems. [25] and [26] addressed the problem of sampled-data MPC for PLPV system in the form of impulsive model. The sampled parameter vector is considered as a new parameter which is the price to pay for imperfect knowledge of the sampled parameter. Also, heavy online computation is required in the proposed methods because a set of LMIs must be solved at each sampling instant to determine the control law. Contrary to the previous approaches, [27] utilized the sampling effect of the scheduling parameter for affine LPV systems where the input matrix does not depend on the parameters. Therefore, this approach cannot be utilized for all class of the PLPV systems. Also, the idea of defining of the system dynamics during the sampling interval inspired in the field of linear impulsive systems [9]. However, applying this approach requires that the parameters vector to be constant during the sampling intervals which is clearly in contradiction with the goal of analyzing the impact of parameters sampling on the stability of the closed-loop system.

Most of the previous studies in the field of sampled-data LPV systems assumed that the parameter vector is constant in between two consecutive samples whereas this assumption is only valid for systems with slowly varying parameters and small sampling periods. Therefore, it can not cover applications such as network control systems with aperiodic sampling. For convenience of study, in some attempts in the literature of sampled-data LPV control systems the sampled parameter is considered as a new parameter which is quite conservative and is a major limitation for improving performance. In addition, the effects of the scheduling parameters sampling on the performance and transient behaviors of the closed loop system is another important subject that needs to be carefully considered. To fill these gaps, in this paper the uncertainty of the measured parameters in the controller are transferred to that of the plant parameters and the closed-loop sampled-data PLPV system is represented by a double-layer polytopic uncertain model. This technique can be applied to 
a large class of multi model systems like Takagi-sugeno(T-S) model [28] which gives an effective way to represent complex nonlinear systems by some simple local linear dynamic systems.

The main contribution of this paper is to present exponential stability analysis and design criteria for nonlinear sampled-data systems under aperiodic samplings based on the PLPV modeling approach. By introducing a new double-layer polytopic formulation for nonlinear sampled-data systems, a candidate discrete Lyapunov-Krasovskii functional is proposed which, by using Wirtingers inequality, provides less conservative robust stability conditions than the existing approaches. Under the new stability criteria, an approach is introduced to synthesize a sampled-data PLPV controller considering some constraints on the location of the closed-loop poles in the presence of uncertainties on the varying parameters.

The paper is organized as follows. Section 2 presents the problem formulation and some assumptions used in this paper. Section 3 presents the derivation of exponential stability condition for sampled-data PLPV systems. In section 4 the problem of pole placement for sampled-data PLPV controller with aperiodic sampling time is stated. Numerical examples and conclusion are given in sections 5 and 6 , respectively.

Notation. Throughout the paper, sets of real numbers, nonnegative real numbers, real vectors of dimension $n$, matrices of dimension $n \times m$, symmetric and symmetric positive definite matrices of dimension $n$ are denoted by $R, R^{+}, R^{n}, R^{n \times m}, S^{n}$ and $S_{+}^{n}$ respectively. $\|$.$\| stands for the Euclidean$ norm of a vector. For any square matrix $A \in R^{n \times n}$, we define $H e(A)=A+A^{T}$. The notation $P>0$, for $P \in R^{n \times n}$, means that $P$ is symmetric and positive definite. For symmetric matrices $X$ and $Y, X<Y$ means that $X-Y$ is negative definite. The notation $I$ and 0 stand for the identity and zero matrices of appropriate dimensions. The symmetric elements of any symmetric matrix denoted by $*$. For a given positive number $T$, let $W:[-T, 0] \rightarrow R^{n}$ be the space of absolutely continuous functions, with square integrable first order derivatives mapping the interval $[-T, 0]$ to $R^{n}$. Consider function $x_{t} \in W$ defined as $x_{t}(\theta)=x(t+\theta), \theta \in[-T, 0]$. Similar to [11], we denote the norm of $x_{t}$ by

$$
\left\|x_{t}\right\|_{W}=\max _{\theta \in[-T, 0]}\left\|x_{t}(\theta)\right\|+\left[\int_{-T}^{0}\left\|\dot{x}_{t}(q)\right\|^{2} d q\right]^{0.5} .
$$

\section{PROBLEM FORMULATION}

Linear parameter varying systems are a class of systems whose state space matrices depend on a time-varying parameter vector $\eta: \mathbb{R}^{+} \rightarrow \mathbb{R}^{n_{\eta}}$. It is assumed that, the parameter vector $\eta(t)$ varies continuously and its derivative is bounded. Define

$$
\Phi:=\left\{\eta: \mathbb{R}^{+} \rightarrow \mathbb{R}^{n_{\eta}} \mid \eta(t) \in \Delta_{\eta}, \dot{\eta}(t) \in \Delta_{v}\right\}
$$

where $\Delta_{\eta}$ and $\Delta_{v}$ are respectively the set of admissible parameter and its derivative. We also assume that the sets $\Delta_{\eta}$ and $\Delta_{v}$ are compact and convex polyhedrons and bounded as follows:

$$
\begin{aligned}
& \Delta_{\eta}=\left\{\eta: R^{+} \rightarrow R^{n_{\eta}} \mid \underline{m}_{i} \leq \eta_{i}(t) \leq \bar{m}_{i}, i=1, \ldots, n_{\eta}\right\} \\
& \Delta_{v}=\left\{\dot{\eta}: R^{+} \rightarrow R^{n_{\eta}} \mid \underline{v}_{i} \leq \dot{\eta}_{i}(t) \leq \bar{v}_{i}, \quad i=1, \ldots, n_{\eta}\right\}
\end{aligned}
$$

The state space model of LPV systems, defined as:

$$
\dot{x}(t)=A(\eta(t)) x(t)+B(\eta(t)) u(t)
$$

where $x(t) \in R^{n_{x}}$ is the state vector and $u(t) \in R^{n_{u}}$ is the control input vector.

In the polytopic representation, the system state-space matrices take values inside a polytope which is the convex hull of the parameters of a set of models, called vertex models. The system 
matrices are given by

$$
A(\eta(t))=\sum_{i=1}^{N} \alpha_{i}(t) A_{i} \quad B(\eta(t))=\sum_{i=1}^{N} \alpha_{i}(t) B_{i} .
$$

where the time-varying parameters are given by $\eta(t)=\sum_{i=1}^{N} \alpha_{i}(t) \Lambda_{i}$ where $\Lambda_{i}$ (vertices of the polytope) are the extremal values of $\eta(t)$ and $\alpha_{i}(t)$ are the time-varying polytopic coordinates evolving over the unit simplex $\Sigma$ which is defined as

$$
\Sigma=\left\{\alpha(t)=\operatorname{col}\left(\alpha_{i}(t)\right): \sum_{i=1}^{N} \alpha_{i}(t)=1, \alpha_{i}(t) \in[0,1], N=2^{n_{\eta}}\right\} .
$$

Any LPV system can be represented, up to a certain degree of accuracy, by a PLPV system. Whenever the matrices of the LPV system are affinely depend on $\eta(t)$, there exists a linear relationship between $\eta(t)$ and $\alpha(t)$ that can be easily determined from the physical model.

As shown in Fig. 1 the controller output at each sampling instant is updated based on the value of the state vector and the corresponding LPV parameter vector, so the control signal will be piecewise constant. We consider the following polytopic state feedback controller:

$$
u(t)=u\left(t_{n}\right)=\sum_{i=1}^{N} \alpha_{i}\left(t_{n}\right) K_{i} x\left(t_{n}\right) \quad t \in\left[t_{n}, t_{n+1}\right), n \in N
$$

where, $K_{i}$ denotes the $i$-th controller gain at the $i$-th vertex of the polytope that need to be determined. Then the closed-loop interconnection of Eq.(4) and Eq.(7) is

$$
\dot{x}(t)=\sum_{i=1}^{N} \alpha_{i}(t) A_{i} x(t)+\sum_{i=1}^{N} \alpha_{i}(t) B_{i} \sum_{i=1}^{N} \alpha_{i}\left(t_{n}\right) K_{i} x\left(t_{n}\right) .
$$

In networked setting, the effect of the network and aperiodic sampling period are integrated in a single input-delay value $\tau(t)$. We model the sampled-data structure as a time-varying delay in the input. The delay induced by the sampler is defined by:

$$
\tau(t)=t-t_{n} \quad \text { for } t \in\left[t_{n}, t_{n+1}\right), n \in N,
$$

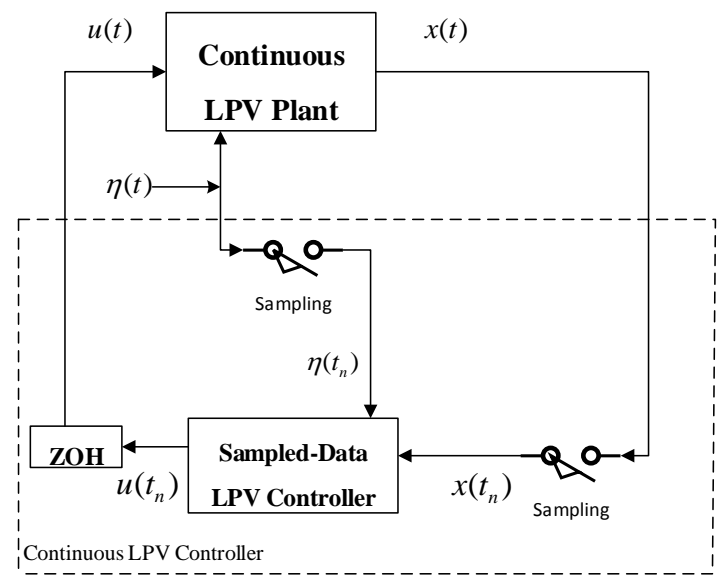

Figure 1. Closed-loop LPV sampled-data system. 
and the largest sampling interval is denoted by

$$
T=\operatorname{Sup}\left(T_{n}\right) .
$$

Where $T_{n}=t_{n+1}-t_{n}$. Difference between the real continuous polytopic parameters of the plant and the piecewise constant polytopic parameters of the controller depends on the sampling interval and the rate of parameter variations. It is difficult to make a correspondence between the derivative of the initial parameters and the derivative of the polytopic parameters. To overcome this difficulty, for known derivative bounds on $\eta(t)$, it is possible to define a polytope that $\dot{\alpha}_{i}(t)$ evolves within it. Based on the bounds on the rate of the parameter variation, we define the following convex polytope containing the parameter derivative.

$$
\dot{\eta}(t)=\sum_{i=1}^{N} \beta_{i}(t) \Gamma_{i}
$$

where $\Gamma_{i}$ are the vertices of the polytope and $\beta_{i}(t)$ are the time-varying polytopic coordinates. Therefore, we have

$$
\sum_{i=1}^{N} \dot{\alpha}_{i}(t) \Lambda_{i}=\sum_{i=1}^{N} \beta_{i}(t) \Gamma_{i}
$$

Combing the equality constraints $\sum_{i=1}^{N} \dot{\alpha}_{i}(t)=0$ and $\sum_{i=1}^{N} \beta_{i}(t)=1$ and (12), we get

$$
\bar{\Lambda}\left[\begin{array}{c}
\dot{\alpha}_{1}(t) \\
\vdots \\
\dot{\alpha}_{N}(t)
\end{array}\right]=\left[\begin{array}{ccc}
\Gamma_{1} & \cdots & \Gamma_{N} \\
1 & \cdots & 1
\end{array}\right]\left[\begin{array}{c}
\beta_{1}(t) \\
\vdots \\
\beta_{N}(t)
\end{array}\right]-\left[\begin{array}{c}
0 \\
1
\end{array}\right], \bar{\Lambda}=\left[\begin{array}{ccc}
\Lambda_{1} & \cdots & \Lambda_{N} \\
1 & \cdots & 1
\end{array}\right]
$$

Let $\Lambda=\left[\Lambda_{1}-\Lambda_{N}, \Lambda_{2}-\Lambda_{N}, \ldots, \Lambda_{N-1}-\Lambda_{N}\right]$. Since $\Lambda \in R^{\log _{2}^{N} \times(N-1)}$, then the row rank of $\Lambda$ is equal to the dimension of the subspace in $R^{N-1}$ spanned by the rows of $\Lambda$. Since $\sum_{i=1}^{N} \alpha_{i}(t)=$ 1 , then, one easily obtains $\eta(t)-\Lambda_{N}=\sum_{i=1}^{N-1} \alpha_{i}(t)\left(\Lambda_{i}-\Lambda_{N}\right)$. This implies that, $\operatorname{rank}(\Lambda)=$ $\operatorname{dim}(\eta(t))=\log _{2}^{N}$. On the other hand, we know that the rank of a matrix is preserved under elementary row operations. Therefore, by applying a set of row operations to $\bar{\Lambda}$, we get:

$$
\widehat{\Lambda}=\left[\begin{array}{cccc}
\Lambda_{1}-\Lambda_{N} & \cdots & \Lambda_{N-1}-\Lambda_{N} & 0 \\
1 & \cdots & 1 & 1
\end{array}\right]
$$

where, $\operatorname{rank}(\bar{\Lambda})=\operatorname{rank}(\widehat{\Lambda})$. Obviously, the equation (14) implies that $\operatorname{rank}(\widehat{\Lambda})=\log _{2}^{N}+1$. As a result, $\bar{\Lambda}$ is a full row rank matrix and as characterized in [29], it has a right pseudoinverse $\bar{\Lambda}^{\dagger}$ such that $\bar{\Lambda} \bar{\Lambda}^{\dagger}=I$. Then equation (13) has solutions in $\dot{\alpha}(t)$ and considering $\beta_{i}(t) \in[0,1]$, we get

$$
\underline{v}_{i} \leq \dot{\alpha}_{i}(t) \leq \bar{v}_{i}, \quad i=1, \ldots ., N
$$

$\dot{\alpha}_{i}(t)$ do not belong to the unitary simplex and will necessarily take negative values since the constraint $\sum_{i=1}^{N} \dot{\alpha}_{i}(t)=0$ must be satisfied.

Using (15) and from mean-value theorem, one gets

$$
\underline{v}_{i} T \leq \alpha_{i}(t)-\alpha_{i}\left(t_{n}\right) \leq \bar{v}_{i} T
$$

So, the following relationship between the measured piecewise constant parameter and the real continuous parameter is obtained:

$$
\alpha_{i}(t)=\alpha_{i}\left(t_{n}\right)+\Delta_{i}(t), \underline{v}_{i} T \leq \Delta_{i}(t) \leq \bar{v}_{i} T
$$


Since, $\sum_{i=1}^{N} \alpha_{i}(t)=1$ and $\sum_{i=1}^{N} \alpha_{i}\left(t_{n}\right)=1$ therefore we get $\sum_{i=1}^{N} \Delta_{i}(t)=0$. Then,by replacing Eq.(17) in Eq.(8) we get

$$
\dot{x}(t)=\sum_{i=1}^{N} \lambda_{i}(t)\left(A_{i}+\Delta_{A}(t)\right) x(t)+\sum_{i=1}^{N} \lambda_{i}(t)\left(B_{i}+\Delta_{B}(t)\right) \sum_{i=1}^{N} \lambda_{i}(t) K_{i} x(t-\tau(t))
$$

Where

$$
\begin{aligned}
& \Delta_{A}(t)=\sum_{j=1}^{N-1} \Delta_{j}(t)\left(A_{j}-A_{N}\right), \Delta_{B}(t)=\sum_{j=1}^{N-1} \Delta_{j}(t)\left(B_{j}-B_{N}\right) \\
& \lambda_{i}(t)=\alpha_{i}\left(t_{n}\right)=\alpha_{i}(t-\tau(t))
\end{aligned}
$$

In this way, the uncertainty on the measured parameters of the controller is turned into the parameters of the plant and the closed-loop sampled-data LPV system represented by a double-layer polytopic description model as illustrated in Fig. 2. The first polytope represents a LPV system in each vertex where the LPV controller is designed for sampled-data of varying parameters shown as nominal vertex $A_{1}, A_{2}, A_{3}, A_{4}$. The second polytop is constructed at each vertex of the system in order to model uncertainty of the varying parameters proportional to sampling period and the rate of parameter variations as shown by $A_{1,1}, A_{1,2} A_{1,3}$ around the first nominal vertex $A_{1}$. It is obvious that the systems(8) and (18) are equivalent. Thus, the stability analysis and design problems of (8) can be transformed to the same problem of (18), that can be easily verified using convex optimization techniques at the vertices of the model. To the best of the authors knowledge, this formulation for sampled-data LPV systems has not already been addressed in the literatures.

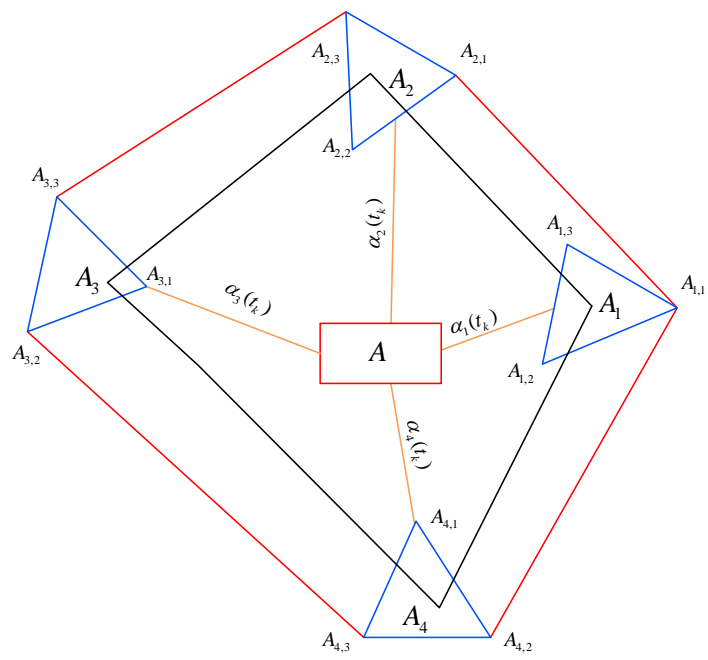

Figure 2. Double-layer polytopic description of PLPV sampled-data system.

\section{EXPONENTIAL STABILITY CRITERIA}

In this section, the problem of exponential stability analysis for system in Eq.(18) is addressed. It is assumed that the feedback gain matrices $K_{j}$ have been well designed. In the following the sufficient conditions are formulated in terms of LMIs to find the MASP that preserves the closedloop system's exponential stability provided that the controller is implemented in a sample-and-hold 
fashion. In the following we decoupled LKF matrix functions and system matrices which allows us to achieve robust exponential stability results. Before starting exponential stability analysis, the following lemmas which will be used in the proofs of our results are presented.

Lemma 1 ([11])

Let there exist positive numbers $\beta, \delta$ and functional $V: R \times W \times L_{2}[-T, 0] \rightarrow R$ such that

$$
\beta\left\|x_{t}(0)\right\|^{2} \leq V\left(t, x_{t}, \dot{x}_{t}\right) \leq \delta\left\|x_{t}\right\|_{W}^{2} .
$$

Let the function $\bar{V}(t)=V\left(t, x_{t}, \dot{x}_{t}\right)$ is continuous from the right for $x_{t}$ satisfying Eq.(18), absolutely continuous for $t \neq t_{n}$ and satisfies $\lim _{t \rightarrow t_{n}^{-}} \bar{V}(t) \geq \bar{V}\left(t_{n}\right)$. If along Eq.(18)

$$
\dot{\bar{V}}(t) \leq-\sigma \bar{V}(t)
$$

then system in Eq.(18) is exponentially stable with the decay rate $\sigma / 2$ and $\mu=\sqrt{\delta / \beta}$.

Lemma 2 ([30])

For matrices $M, N$ and $R>0$ with appropriate dimensions, the following inequality holds.

$$
M N+N^{T} M^{T} \leq M R M^{T}+N^{T} R^{-1} N
$$

Lemma 3 ([31])

(Wirtinger inequality) For a given matrix $R \in S_{+}^{n}$, the following inequality holds for all continuously differentiable function $x \in[a, b] \rightarrow R^{n}$ :

$$
\int_{a}^{b} \dot{x}^{T}(t) R \dot{x}(t) d t \geq \frac{1}{b-a}(x(b)-x(a))^{T} R(x(b)-x(a))+\frac{3}{b-a} \tilde{\Omega}^{T} R \tilde{\Omega}
$$

Where $\tilde{\Omega}=x(b)+x(a)-\frac{2}{b-a} \int_{a}^{b} x(t) d t$.

The next theorem provides conditions for exponential stability of the PLPV system in the Eq.(18). In some cases, for the sake of simplicity, explicit dependence on $t$ omitted to shorten the notation.

\section{Theorem 1}

The PLPV system in Eq.(18) with aperiodic sampling intervals smaller than $T>0$ is exponentially stable if there exist $P \in S_{+}^{n}, X, M_{11}, M_{22} \in S^{n}, H_{i j} \in R^{3 n}, Q_{i j} \in R^{5 n}$ with $H_{i j}=H_{j i}{ }^{T}, Q_{i j}=$ $Q_{j i}{ }^{T}$ and matrices $X_{1}, M_{12}, F_{11, i}, F_{12, i}, F_{13, i}, F_{21, i}, F_{22, i}, F_{23, i} \in R^{n \times n}$ such that the following LMIs hold for $i, j=1,2, \ldots, N$.

$$
\begin{aligned}
& M=\left[\begin{array}{cc}
M_{11} & M_{12} \\
* & M_{22}
\end{array}\right]>0 \\
& {\left[\begin{array}{ll}
I & 0
\end{array}\right]^{T} P\left[\begin{array}{ll}
I & 0
\end{array}\right]+T\left[\begin{array}{cc}
X & -X+X_{1} \\
-X+X_{1}^{T} & X-X_{1}-X_{1}^{T}
\end{array}\right]>0} \\
& \Pi_{i j}+\Pi_{j i}+T\left(\Phi_{i j}+\Phi_{j i}\right)<H_{i j}+H_{j i} \\
& {\left[\begin{array}{ccc}
\Pi_{i j}+\Pi_{j i}+2 T \Theta & T F_{1, i}+T F_{1, j} & T F_{2, i}+T F_{2, j} \\
* & -2 T M_{11} & 0 \\
* & * & -6 T M_{11}
\end{array}\right]<Q_{i j}+Q_{j i}} \\
& {\left[\begin{array}{ccc}
H_{11} & \cdots & H_{1 N} \\
\vdots & \ddots & \vdots \\
H_{N 1} & \cdots & H_{N N}
\end{array}\right]<0, \quad\left[\begin{array}{ccc}
Q_{11} & \cdots & Q_{1 N} \\
\vdots & \ddots & \vdots \\
Q_{N 1} & \cdots & Q_{N N}
\end{array}\right]<0}
\end{aligned}
$$


Where

$$
\begin{aligned}
& \Pi_{i j}(1,1)=H e\left(P\left(A_{i}+\Delta_{A}(t)\right)-F_{11, i}-3 F_{21, i}\right)-X \\
& \Pi_{i j}(1,2)=P\left(B_{i}+\Delta_{B}(t)\right) K_{j}-X_{1}+X+F_{11, i}-F_{12, i}^{T}-3 F_{21, i}-3 F_{22, i}^{T}-M_{12} \\
& \Pi_{i j}(1,3)=-F_{13, i}^{T}+6 F_{21, i}-3 F_{23, i}^{T} \\
& \Pi_{i j}(2,2)=-X+H e\left(X_{1}+F_{12, i}-3 F_{22, i}^{T}+M_{12}\right) \\
& \Pi_{i j}(2,3)=F_{13, i}^{T}+6 F_{22, i}-3 F_{23, i}^{T}, \quad \Pi_{i j}(3,3)=6 F_{23, i}+6 F_{23, i}^{T} \\
& \Theta=-\left[\begin{array}{lll}
0 & I & 0
\end{array}\right]^{T} M_{22}\left[\begin{array}{ccc}
0 & I & 0
\end{array}\right] \\
& \Phi_{i j}(1,1)=H e\left(X\left(A_{i}+\Delta_{A}(t)\right)\right)+\left(A_{i}^{T}+\Delta_{A}^{T}(t)\right) M_{11}\left(A_{i}+\Delta_{A}(t)\right) \\
& \Phi_{i j}(1,2)=\left(A_{i}^{T}+\Delta_{A}^{T}(t)\right)\left(X_{1}-X+M_{12}+M_{12}\left(B_{i}+\Delta_{B}(t)\right) K_{j}\right)+X\left(B_{i}+\Delta_{B}(t)\right) K_{j} \\
& \Phi_{i j}(2,2)=H e\left(\left(X_{1}^{T}-X+M_{12}^{T}\right)\left(B_{i}+\Delta_{B}(t)\right) K_{j}\right)+ \\
& M_{22}+K_{j}^{T}\left(B_{i}^{T}+\Delta_{B}^{T}(t)\right) M_{11}\left(B_{i}+\Delta_{B}(t)\right) K_{j} \\
& \Phi_{i j}(1,3)=\Phi_{i j}(2,3)=\Phi_{i j}(3,3)=0
\end{aligned}
$$

Proof

At the first step, we consider the following LKF for exponential stability analysis:

$$
\begin{aligned}
\bar{V}(t) & =V_{1}(t)+V_{2}\left(t, x_{t}, \dot{x}_{t}\right)+V_{3}\left(t, x_{t}\right) \quad t \in\left[t_{n}, t_{n+1}\right) \\
V_{1}(t) & =x^{T}(t) P x(t) \\
V_{2}(t) & =\left(t_{n+1}-t\right) \int_{t_{n}}^{t}\left[\begin{array}{c}
\dot{x}(q) \\
x\left(t_{n}\right)
\end{array}\right]^{T} M\left[\begin{array}{c}
\dot{x}(q) \\
x\left(t_{n}\right)
\end{array}\right] d q \\
V_{3}(t) & =\left(t_{n+1}-t\right) \xi^{T}(t)\left[\begin{array}{cc}
X & -X+X_{1} \\
-X+X_{1}^{T} & X-X_{1}-X_{1}^{T}
\end{array}\right] \xi(t)
\end{aligned}
$$

Where $\xi^{T}(t)=\left[x^{T}(t), x^{T}\left(t_{n}\right)\right]$.

We utilized, a piecewise smooth LKF which decreases the conservatism of the stability conditions when compared with a smooth LKF used for input delay approaches in [24, 32]. Performance of LKF is intuitive, therefore we will show that the proposed structure lead to less conservative stability conditions. Based on Lemma 1, the candidate LKF must be right continuous. In the following lemma, right continuity property of the LKF in Eq.(25) is presented.

\section{Lemma 4}

The KFL defined in Eq.(25) is a right continuous and non increasing function of time at sampling instant $t_{n}$.

\section{Proof}

We assume that decision matrix $P, M, X, X_{1}$ are chosen in such a way that condition in Eq.(20) satisfied. Obviously, the Lyapunov function $V_{1}$ has quadratic form and thus is continuous. $V_{2}$ is continuous between two sampling instants, so they are non-negative at $t_{n}^{-}$. At instant $t_{n}$, the lower and upper bounds of the integral become equal, thus $V_{2}$ get zero. Also, $V_{3}$ is continuous between two sampling instants and is non-negative at $t_{n}^{-}$. At the instants $t_{n}$, because of $x(t)=x\left(t_{n}\right), V_{3}$ get zero. Therefore, the condition

$$
\lim _{t \rightarrow t_{n}^{-}} \bar{V}(t) \geq \bar{V}\left(t_{n}\right)
$$

holds.

After sampling, since $\lim _{t \rightarrow t_{n}+} \tau(t)=\tau\left(t_{n}\right)=0$ then $t=t_{n}$, and we have

$$
\lim _{t \rightarrow t_{n}+} V_{2}(t)+V_{3}(t)=0 .
$$

Thus $\bar{V}(t)$ is right continuous in time since $\lim _{t \rightarrow t_{n^{+}}} \bar{V}(t)=\bar{V}\left(t_{n}\right)$. 
In the following, we compute lower and upper bounds of candidate LKF to ensure that inequality in Eq.(20) satisfied. Constraint (24a) is sufficient conditions for positiveness of integral terms. By combination non-integral terms, we get

$$
\begin{aligned}
& V_{1}+V_{3}=\xi^{T}(t) \Psi \xi(t), \quad t \in\left[t_{n}, t_{n+1}\right) \\
& \Psi=\left[\begin{array}{ll}
I & 0
\end{array}\right]^{T} P\left[\begin{array}{ll}
I & 0
\end{array}\right]+\left(t_{n+1}-t\right)\left[\begin{array}{cc}
X & -X+X_{1} \\
-X+X_{1}^{T} & X-X_{1}-X_{1}^{T}
\end{array}\right]
\end{aligned}
$$

Since $P>0$ and Eq.(24b) are sufficient condition for existence small $\beta$ such that

$$
\beta\|x(t)\|^{2}=\beta\left\|x_{t}(0)\right\|^{2} \leq \bar{V}(t) .
$$

Using the inequalities $\|x(t)\| \leq\left\|x_{t}\right\|_{W}$ and $\|\xi(t)\| \leq \sqrt{2}\|x(t)\|$ in Eq.(28), one gets

$$
V_{1}(t)+V_{3}(t) \leq 2 \lambda_{\max }(\Psi)\left\|x_{t}\right\|_{W}^{2} .
$$

Also for $V_{2}(t)$, we have

$$
V_{2} \leq T \lambda_{\max }(M)\left(\int_{t_{n}}^{t}\|\dot{x}(q)\|^{2} d q+\int_{t_{n}}^{t}\left\|x\left(t_{n}\right)\right\|^{2} d q\right)
$$

Considering $\int_{-T}^{0}\left\|\dot{x}_{t}(q)\right\|^{2} d q \leq\left\|x_{t}\right\|_{W}^{2}$ from Eq.(1) we can write

$$
V_{2} \leq T \lambda_{\max }(M)(1+T)\left\|x_{t}\right\|_{W}^{2}
$$

Adding inequalities in Eq.(30) and Eq.(32) the upper bound on $\bar{V}(t)$ is obtained as

$$
\bar{V}(t) \leq\left\{2 \lambda_{\max }(\Psi)+T\left(\lambda_{\max }(M)\right)(1+T)\right\}\left\|x_{t}\right\|_{W}^{2} .
$$

Consequently, inequalities in Eqs.(29) and (33) guarantee the condition in Eq.(20).

The exponential stability and stabilization conditions are derived from the time derivative of LKF. Therefore candidate LKF must satisfy inequalities in Eq.(21). The derivative of Lyapunov function $V_{1}(t)$ along the system trajectory is given by

$$
\begin{aligned}
& \dot{V}_{1}=x^{T}\left(\sum_{i=1}^{N} \lambda_{i} H e\left(P\left(A_{i}+\Delta_{A}(t)\right)\right)\right) x+x^{T}\left(t_{n}\right)\left(\sum_{i, j=1}^{N} \lambda_{i} \lambda_{j} P\left(B_{i}+\Delta_{B}(t)\right) K_{j}\right) x \\
& +x^{T}\left(\sum_{i, j=1}^{N} \lambda_{i} \lambda_{j} K_{j}^{T}\left(B_{i}+\Delta_{B}(t)\right)^{T} P\right) x\left(t_{n}\right)
\end{aligned}
$$

Also the derivative of Lyapunov function $\dot{V}_{3}\left(t, x_{t}\right)$ along the system trajectory is given by

$$
\begin{aligned}
& \dot{V}_{3}=\xi^{T}\left[\begin{array}{cc}
X & -X+X_{1} \\
* & X-X_{1}-X_{1}^{T}
\end{array}\right] \xi+ \\
& \left(t_{n+1}-t\right) \xi^{T}\left(\sum_{i=1}^{N} \lambda_{i}\left[\begin{array}{cc}
H e\left(X\left(A_{i}+\Delta_{A}(t)\right)\right) & \left(A_{i}+\Delta_{A}(t)\right)^{T} \\
* & 0
\end{array}\right]\right) \xi+ \\
& \left(t_{n+1}-t\right) \xi^{T}\left(\sum_{i, j=1}^{N} \lambda_{i} \lambda_{j}\left[\begin{array}{cc}
0 & X\left(B_{i}+\Delta_{B}(t)\right) K_{j} \\
* & H e\left(\left(-X+X_{1}\right)\left(B_{i}+\Delta_{B}(t)\right) K_{j}\right)
\end{array}\right]\right) \xi
\end{aligned}
$$

Differentiating $V_{2}\left(t, x_{t}, \dot{x}_{t}\right)$ along the system trajectory, we get

$$
\begin{aligned}
& \dot{V}_{2}=\left(t_{n+1}-t\right)\left\{\dot{x}^{T}(t) M_{11} \dot{x}(t)+x^{T}\left(\sum_{i=1}^{N} \lambda_{i} 2\left(A_{i}+\Delta_{A}(t)\right)^{T} M_{12}\right) x\left(t_{n}\right)\right\} \\
& +\left(t_{n+1}-t\right)\left\{x^{T}\left(t_{n}\right) \sum_{i, j=1}^{N} \lambda_{i} \lambda_{j} 2\left(\left(B_{i}+\Delta_{B}(t)\right) K_{j}\right)^{T} M_{12} x\left(t_{n}\right)+x^{T}\left(t_{n}\right) M_{22} x\left(t_{n}\right)\right\} \\
& -\int_{t_{n}}^{t} \dot{x}^{T}(q) M_{11} \dot{x}(q) d q-2\left(x(t)-x\left(t_{n}\right)\right) M_{12} x\left(t_{n}\right)-\tau x^{T}\left(t_{n}\right) M_{22} x\left(t_{n}\right)
\end{aligned}
$$


Since $M_{11}$ is positive definite, it can be rewritten as $M_{11}=M_{11}^{0.5} M_{11}^{0.5}$. Using Eq.(18), we get

$$
\left.\begin{array}{l}
\dot{x}^{T} M_{11} \dot{x}=0.5 \sum_{i, j, n, l=1}^{N} \lambda_{i} \lambda_{j} \lambda_{n} \lambda_{l}\left(\xi ^ { T } [ \begin{array} { c } 
{ A _ { i } ^ { T } + \Delta _ { A } ^ { T } } \\
{ K _ { j } ^ { T } ( B _ { i } ^ { T } + \Delta _ { B } ^ { T } ) }
\end{array} ] M _ { 1 1 } ^ { 0 . 5 } M _ { 1 1 } ^ { 0 . 5 } \left[A_{l}+\Delta_{A}\right.\right. \\
\left.\left.+0.5 \sum_{i, j, n, l=1}^{N} \lambda_{i} \lambda_{j} \lambda_{n} \lambda_{l}\left(B_{l}+\Delta_{B}\right) K_{n}\right] \xi\right) \\
\xi_{l}^{T}+\Delta_{A}^{T} \\
K_{n}^{T}\left(B_{l}^{T}+\Delta_{B}^{T}\right)
\end{array}\right] M_{11}^{0.5} M_{11}^{0.5}\left[\begin{array}{ll}
A_{i}+\Delta_{A} & \left.\left.\left(B_{i}+\Delta_{B}\right) K_{j}\right] \xi\right)
\end{array}\right.
$$

Applying Lemma 2 on (37), with considering

$$
\begin{aligned}
& N=M_{11}^{0.5}\left[A_{l}+\Delta_{A} \quad\left(B_{l}+\Delta_{B}\right) K_{n}\right] \\
& M^{T}=M_{11}^{0.5}\left[\begin{array}{ll}
A_{i}+\Delta_{A} & \left(B_{i}+\Delta_{B}\right) K_{j}
\end{array}\right], R=I
\end{aligned}
$$

the following inequality obtained.

$$
\dot{x}^{T} M_{11} \dot{x}<\sum_{i, j=1}^{N} \lambda_{i} \lambda_{j} \xi^{T}\left[\begin{array}{cc}
\left(A_{i}^{T}+\Delta_{A}^{T}\right) M_{11}\left(A_{i}+\Delta_{A}\right) & \left(A_{i}^{T}+\Delta_{A}^{T}\right) M_{11}\left(B_{i}+\Delta_{B}\right) K_{j} \\
* & \left(K_{j}^{T}\left(B_{i}^{T}+\Delta_{B}^{T}\right)\right) M_{11}\left(B_{i}+\Delta_{B}\right) K_{j}
\end{array}\right] \xi
$$

By defining vector $\zeta(t)$ as

$$
\zeta(t)=\left[\begin{array}{lll}
x(t) & x\left(t_{n}\right) & \frac{\int_{t_{n}}^{t} x(q) d q}{\tau(t)}
\end{array}\right]
$$

and using Lemma (3), we have

$$
\begin{aligned}
& -\int_{t_{n}}^{t} \dot{x}^{T}(q) M_{11} \dot{x}(q) d q \leq-\frac{2}{\tau(t)} \zeta^{T}\left(W_{1} M_{11} W_{1}^{T}+3 W_{2} M_{11} W_{2}^{T}\right) \zeta \\
& W_{1}^{T}=\left[\begin{array}{lll}
I & -I & 0
\end{array}\right], \quad W_{2}^{T}=\left[\begin{array}{lll}
I & I & -2 I
\end{array}\right]
\end{aligned}
$$

By using Lemma. (2), the following inequalities replaced in Eq(41)

$$
\begin{aligned}
& F_{1}(\lambda) W_{1}^{T}+W_{1} F_{1}^{T}(\lambda) \leq \frac{2}{\tau(t)} W_{1} M_{11} W_{1}^{T}+\frac{\tau(t)}{2} F_{1}(\lambda) M_{11}^{-1} F_{1}^{T}(\lambda) \\
& F_{2}(\lambda) W_{2}^{T}+W_{2} F_{2}^{T}(\lambda) \leq \frac{2}{\tau(t)} W_{2} M_{11} W_{2}^{T}+\frac{\tau(t)}{2} F_{2}(\lambda) M_{11}^{-1} F_{2}^{T}(\lambda) \\
& F_{1}^{T}(\lambda)=\sum_{i=1}^{N} \lambda_{i}\left[\begin{array}{llll}
F_{11, i}^{T} & F_{12, i}^{T} & F_{13, i}^{T}
\end{array}\right], F_{2}(\lambda)=\sum_{i=1}^{N} \lambda_{i}\left[\begin{array}{lll}
F_{21, i}^{T} & F_{22, i}^{T} & F_{23, i}^{T}
\end{array}\right]
\end{aligned}
$$

Therefore $\dot{\bar{V}}(t)$ yields

$$
\begin{aligned}
& \dot{\bar{V}}(t)=\dot{V}_{1}(t)+\dot{V}_{2}(t)+\dot{V}_{3}(t) \leq \sum_{i, j=1}^{N} \lambda_{i} \lambda_{j} \zeta^{T}\left(\Pi_{i j}+(T-\tau) \Phi_{i j}+\tau \Theta\right) \zeta+ \\
& \tau \zeta^{T}\left(F_{1} M_{11}^{-1} F_{1}^{T}+3 F_{2} M_{22}^{-1} F_{2}^{T}\right) \zeta
\end{aligned}
$$

For $\tau=0$, Eq.(24c) and Eq.(24e) implies

$$
\begin{gathered}
\dot{\bar{V}}(t) \leq \zeta^{T}\left(\sum_{i=1}^{N} \lambda_{i}{ }^{2}\left(\Pi_{i i}+T \Phi_{i i}\right)+\sum_{i=1}^{N} \sum_{i<j}^{N} \lambda_{i} \lambda_{j}\left(\Pi_{i j}+\Pi_{j i}+T\left(\Phi_{i j}+\Phi_{j i}\right)\right)\right) \zeta< \\
{\left[\begin{array}{c}
\lambda_{1} \zeta \\
\vdots \\
\lambda_{N} \zeta
\end{array}\right]^{T}\left[\begin{array}{ccc}
H_{11} & \cdots & H_{1 N} \\
\vdots & \ddots & \vdots \\
H_{N 1} & \cdots & H_{N N}
\end{array}\right]\left[\begin{array}{c}
\lambda_{1} \zeta \\
\vdots \\
\lambda_{N} \zeta
\end{array}\right]<-\alpha_{1} \sum_{i=1}^{N} \lambda_{i}{ }^{2} \zeta^{T} \zeta<-\alpha_{1} \zeta^{T} \zeta}
\end{gathered}
$$


Also for $\tau=T$, by applying Schur complement to Eq.(43) thus Eq.(24d) and Eq.(24e) implies

$$
\begin{aligned}
& \dot{\bar{V}}(t) \leq \bar{\zeta}^{T}\left(\sum_{i=1}^{N} \lambda_{i}^{2}\left[\begin{array}{ccc}
\Pi_{i i}+T \Theta & T F_{1, i} & T F_{2, i} \\
* & -T M_{11} & 0 \\
* & * & -3 T M_{11}
\end{array}\right]+\right. \\
& \left.\sum_{i=1}^{N} \sum_{i<j}^{N} \lambda_{i} \lambda_{j}\left[\begin{array}{ccc}
\Pi_{i j}+\Pi_{j i}+2 T \Theta & T F_{1, i}+T F_{1, j} & T F_{2, i}+T F_{2, j} \\
* & -2 T M_{11} & 0 \\
* & * & -6 T M_{11}
\end{array}\right]\right) \bar{\zeta}<
\end{aligned}
$$

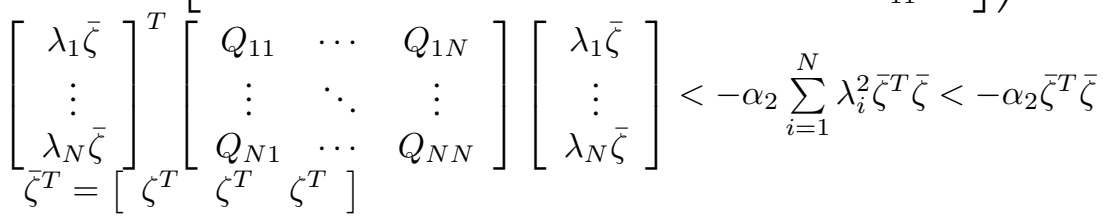

Where $\alpha_{1}>0, \alpha_{2}>0$. Since Eq.(43) is affine in $\tau$, then there exists a small scalar $c>0$ such that $\dot{\bar{V}}(t)<-c\left\|x_{t}\right\|_{W}^{2}$ to hold for any instants $t \in\left[t_{n}, t_{n+1}\right) \cdot \dot{\bar{V}}(t)<-c\left\|x_{t}\right\|_{W}^{2}$ using Eq.(20) implied $\dot{\bar{V}}(t)<-c / \delta \bar{V}(t)$. Then system in Eq.(18) is exponentially stable with the decay rate $c / 2 \delta$. With this completes the proof.

\section{Remark 1}

Here we could extend the stability conditions of LIT sampled-data systems given in [11] and [33] to the PLPV systems described in Eq.(8). However, since $\alpha(t)$ and $\alpha\left(t_{n}\right)$ can take arbitrary values, then the feasibility of the derived conditions must be checked in all subsystems and this will be highly conservative. To moderate this conservatism, an approach is proposed in (44) and (45) to address the interactions of the subsystems via a single matrix and thereby the conservatism due to the interactions is reduced. This is the advantage of extracting the stability conditions of the PLPV sampled-data system in (18). On the other hand, the uncertainty considered in this model is time-varying and thus the stability conditions of the LIT sampled-data systems can not be trivially extended to the PLPV systems described in (18).

\section{Remark 2}

The input-delay approach suffers from an important problem related to the structure of LKF, i.e. it is not clear how to choose the Lyapunov functional. Recently, in some application of sampleddata control for linear systems with uncertainties [32, 34, 35] and LPV systems [24, 22, 23], the stability conditions are derived using a smooth LKF which then utilised in the stability analysis of the systems with fast varying input delays. In this paper, the stability conditions are derived based on a modified form of a piecewise smooth LKF presented in [11]. It is shown that the proposed approach provides less conservative results for sampled-data systems, since it takes the information about the particularity of the sampling-induced saw-tooth delay into account.

\section{Remark 3}

Regarding the proposed LKF in (25), the second component is a functional penalizing the derivative of the state vector and the sampled state vector in the interval $\left[t_{n}, t\right)$. Therefore, we added decision matrices $M_{12}$ and $M_{22}$ into the stability conditions. However, the LKF proposed in [11,33] only considers the derivative of the state vector. Therefore, the proposed LKF in this paper is more general than the existing ones. Furthermore, extra terms $M_{12}, M_{22}, F_{1}$ and $F_{2}$ are added to relax the conditions for ensuring the negativeness of the derivative of the LKF. Therefore, the derived stability conditions are more general than ones used in [11,33] and it gives less conservative stability conditions for sampled-data systems. It is also noted that, the Wirtingers inequalities provide tighter bounds for the functional's time derivative rather than the Jensens inequality used in [11, 34, 35, 33].

The principle of the polytopic formulation is based on the fact that the system and stability conditions have affine dependency on the parameters. If the affine dependency is lost the stability of the system is not equivalent to the feasibility of the LMI at each vertex. In the following some sufficient conditions are expressed to relax the parameter dependent LMI conditions where the affine dependency is lost. 
Lemma 5 ([36])

(Projection Lemma) Given a symmetric matrix $\Psi$ and two matrices $\Gamma$ and $\Sigma$ the problem

$$
\Psi+\Gamma^{T} N \Sigma+\Sigma^{T} N^{T} \Gamma<0
$$

is solvable with respect to decision matrix $N$ if only if

$$
\Gamma^{\perp^{T}} \Psi \Gamma^{\perp}<0 \quad \Sigma^{\perp^{T}} \Psi \Sigma^{\perp}<0
$$

where $\Gamma^{\perp}$ and $\Sigma^{\perp}$ denote arbitrary base of the null spaces of $\Gamma$ and $\Sigma$ respectively, $\Gamma \Gamma^{\perp}=0$ and $\Sigma \Sigma^{\perp}=0$.

The next theorem states the condition for parameter dependent exponential stability of the PLPV system described in Eq.(18).

Theorem 2

The PLPV system in the Eq.(18) with aperiodic sampling intervals smaller than $T>0$ is exponentially stable if there exist $P_{i} \in S_{+}^{n}, X_{i}, M_{11, i}, M_{22, i} \in S^{n}, Y_{i j} \in R^{4 n}, Z_{i j} \in R^{6 n}$ with $Y_{i j}=$ $Y_{j i}{ }^{T}, Z_{i j}=Z_{j i}{ }^{T}$ and matrices $X_{1, i}, N_{1}, N_{2}, N_{3}, N_{4}, M_{12, i}, F_{11, i}, F_{12, i}, F_{13, i}, F_{21, i}, F_{22, i}, F_{23, i} \in$ $R^{n \times n}$ such that the following LMIs hold for $i, j=1,2, \ldots, N$.

$$
\begin{aligned}
& M_{i}=\left[\begin{array}{cc}
M_{11, i} & M_{12, i} \\
* & M_{22, i}
\end{array}\right]>0 \\
& {\left[\begin{array}{cc}
I & 0
\end{array}\right]^{T} P_{i}\left[\begin{array}{ll}
I & 0
\end{array}\right]+T\left[\begin{array}{cc}
X_{i} & -X_{i}+X_{1, i} \\
* & X_{i}-X_{1, i}-X_{1, i}^{T}
\end{array}\right]>0} \\
& \mathrm{E}_{\mathrm{ij}}+\mathrm{E}_{\mathrm{ji}}<Y_{i j}+Y_{j i} \\
& \Xi_{i j}+\Xi_{j i}<Z_{i j}+Z_{j i} \\
& {\left[\begin{array}{ccc}
Y_{11} & \cdots & Y_{1 N} \\
\vdots & \ddots & \vdots \\
Y_{N 1} & \cdots & Y_{N N}
\end{array}\right]<0,\left[\begin{array}{ccc}
Z_{11} & \cdots & Z_{1 N} \\
\vdots & \ddots & \vdots \\
Z_{N 1} & \cdots & Z_{N N}
\end{array}\right]<0}
\end{aligned}
$$

Where

$$
\begin{aligned}
& \mathrm{E}_{\mathrm{ij}}=\left[\begin{array}{cccc}
T M_{11, i}-H e\left(N_{1}\right) & \Lambda_{7, i} & \Lambda_{9, i}+N_{1}^{T}\left(B_{i}+\Delta_{B}(t)\right) K_{j} & -T M_{12, i}+N_{1}^{T} \\
* & \Lambda_{8, i} & \Lambda_{2, i}+N_{2}^{T}\left(B_{i}+\Delta_{B}(t)\right) K_{j} & -P_{i}-T X_{i}+\Lambda_{3, i}+N_{2}^{T} \\
* & * & T M_{22, i}+\Lambda_{4, i} & \Lambda_{9, i}+\Lambda_{5, i} \\
* & * & * & T M_{11, i}+\Lambda_{6, i}
\end{array}\right] \\
& \Xi_{i j}=\left[\begin{array}{cccccc}
-H e\left(N_{3}\right) & \Lambda_{10, i} & N_{3}^{T}\left(B_{i}+\Delta_{B}(t)\right) K_{j} & N_{3}^{T} & 0 & 0 \\
* & \Lambda_{11, i} & \Lambda_{2, i}+N_{4}^{T}\left(B_{i}+\Delta_{B}(t)\right) K_{j} & -P_{i}+\Lambda_{3, i} & T F_{11, i} & 3 T F_{21, i} \\
* & * & T M_{22, i}+\Lambda_{4, i} & \Lambda_{5, i} & T F_{12, i} & 3 T F_{22, i} \\
* & * & * & \Lambda_{6, i} & T F_{13, i} & 3 T F_{23, i} \\
* & * & * & * & -T M_{11, i} & 0 \\
* & * & * & * & * & -3 T M_{11, i}
\end{array}\right] \\
& \Lambda_{1, i}=-X_{i}+H e\left(-F_{11, i}-3 F_{21, i}\right) \\
& \Lambda_{2, i}=-X_{1, i}+X_{i}+F_{11, i}-F_{12, i}^{T}-3 F_{21, i}-3 F_{22, i}^{T}-M_{12, i} \\
& \Lambda_{3, i}=-F_{13, i}^{T}+6 F_{21, i}-3 F_{23, i}^{T}, \quad \Lambda_{4, i}=-X_{i}+H e\left(X_{1, i}+F_{12, i}-3 F_{22, i}^{T}+M_{12, i}\right) \\
& \Lambda_{5, i}=F_{13, i}^{T}+6 F_{22, i}-3 F_{23, i}^{T}, \Lambda_{6, i}=6 F_{23, i}+6 F_{23, i}^{T} \\
& \Lambda_{7, i}=P_{i}+T X_{i}+N_{1}^{T}\left(A_{i}+\Delta_{A}(t)\right)-N_{2}, \Lambda_{8, i}=\Lambda_{1, i}+H e\left(N_{2}^{T}\left(A_{i}+\Delta_{A}(t)\right)\right) \\
& \Lambda_{9, i}=T\left(X_{1, i}-X_{i}-M_{12, i}\right), \Lambda_{10, i}=P_{i}+N_{3}^{T}\left(A_{i}+\Delta_{A}(t)\right)-N_{4} \\
& \Lambda_{11, i}=\Lambda_{1, i}+\operatorname{He}\left(N_{4}^{T}\left(A_{i}+\Delta_{A}(t)\right)\right)
\end{aligned}
$$




\section{Proof}

Following a similar treatment shown as in (44) and (45), when (48c), (48d) and (48e) hold for $i, j=1, \ldots, N$, the following is satisfied

$$
\begin{aligned}
& \sum_{i, j=1}^{N} \lambda_{i}(t) \lambda_{j}(t) \mathrm{E}_{\mathrm{ij}}<0 \\
& \sum_{i, j=1}^{N} \lambda_{i}(t) \lambda_{j}(t) \Xi_{i j}<0
\end{aligned}
$$

Let rewrite (49) as

$$
\sum_{i=1}^{N} \lambda_{i}(t) \Psi_{1, i}+\Gamma_{1}^{T} N \Sigma_{1}+\Sigma_{1}^{T} N^{T} \Gamma_{1}<0
$$

with

$$
\begin{aligned}
\Psi_{1, i} & =\left[\begin{array}{cccc}
T M_{11, i} & P_{i}+T X_{i} & \Lambda_{7, i} & -T M_{12, i} \\
* & \Lambda_{1, i} & \Lambda_{2, i} & -P_{i}-T X_{i}+\Lambda_{3, i} \\
* & * & T M_{22, i}+\Lambda_{4, i} & \Lambda_{7, i}+\Lambda_{5, i} \\
* & * & * & T M_{11, i}+\Lambda_{6, i}
\end{array}\right] \\
\Gamma_{1} & =\left[\begin{array}{cccc}
-I & \sum_{i=1}^{N} \lambda_{i}\left(A_{i}+\Delta_{A}(t)\right) & \sum_{i, j=1}^{N} \lambda_{i} \lambda_{j}\left(B_{i}+\Delta_{B}(t)\right) K_{j} & I
\end{array}\right] \\
\Sigma_{1} & =\left[\begin{array}{llll}
I & 0 & 0 & 0 \\
0 & 0 & I & 0
\end{array}\right], N=\left[\begin{array}{lll}
N_{1} & N_{2}
\end{array}\right]
\end{aligned}
$$

and rewrite (50) as

$$
\sum_{i=1}^{N} \lambda_{i}(t) \Psi_{2, i}+\Sigma_{2}^{T} \bar{N} \Upsilon_{2}+\Upsilon_{2}^{T} \bar{N}^{T} \Sigma_{2}<0
$$

with

$$
\begin{aligned}
\Psi_{2, i} & =\left[\begin{array}{cccccc}
0 & P_{i} & 0 & 0 & 0 & 0 \\
* & \Lambda_{1, i} & \Lambda_{2, i} & -P_{i}+\Lambda_{3, i} & T F_{11, i} & 3 T F_{21, i} \\
* & * & T M_{22, i}+\Lambda_{4, i} & \Lambda_{5, i} & T F_{12, i} & 3 T F_{22, i} \\
* & * & * & \Lambda_{6, i} & T F_{13, i} & 3 T F_{23, i} \\
* & * & * & * & -T M_{11, i} & 0 \\
* & * & * & * & * & -3 T M_{11, i}
\end{array}\right] \\
\Gamma_{2} & =\left[\begin{array}{cccccc}
-I & \sum_{i=1}^{N} \lambda_{i}\left(A_{i}+\Delta_{A}(t)\right) & \sum_{i, j=1}^{N} \lambda_{i} \lambda_{j}\left(B_{i}+\Delta_{B}(t)\right) K_{j} & I & 0 & 0
\end{array}\right] \\
\Sigma_{2} & =\left[\begin{array}{cccccc}
I & 0 & 0 & 0 & 0 & 0 \\
0 & I & 0 & 0 & 0 & 0
\end{array}\right], \bar{N}=\left[\begin{array}{lll}
N_{3} & N_{4}
\end{array}\right]
\end{aligned}
$$


The matrix variables $N_{1}, N_{2}, N_{3}$ and $N_{4}$ are the slack variables. By finding explicit basis for the null-space of $\Gamma_{1}$ and $\Gamma_{2}$ as

$$
\begin{aligned}
\Gamma_{1}^{\perp} & =\left[\begin{array}{ccc}
\sum_{i=1}^{N} \lambda_{i}\left(A_{i}+\Delta_{A}(t)\right) & \sum_{i, j=1}^{N} \lambda_{i} \lambda_{j}\left(B_{i}+\Delta_{B}(t)\right) K_{j} & I \\
I & 0 & 0 \\
0 & I & 0 \\
0 & 0 & I
\end{array}\right] \\
\Gamma_{2}^{\perp} & =\left[\begin{array}{ccccc}
\sum_{i=1}^{N} \lambda_{i}\left(A_{i}+\Delta_{A}(t)\right) & \sum_{i, j=1}^{N} \lambda_{i} \lambda_{j}\left(B_{i}+\Delta_{B}(t)\right) K_{j} & I & 0 & 0 \\
I & 0 & 0 & 0 & 0 \\
0 & I & 0 & 0 & 0 \\
0 & 0 & I & 0 & 0 \\
0 & 0 & 0 & I & 0 \\
0 & 0 & 0 & 0 & I
\end{array}\right]
\end{aligned}
$$

and applying the projection lemma, with considering $P=P_{i}, X=X_{i}, X_{1}=X_{1, i}, \quad M_{11}=$ $M_{11, i}, M_{12}=M_{12, i}, M_{22}=M_{22, i}$ for $i=1, \ldots, N$, we have

$$
\sum_{i, j=1}^{N} \lambda_{i} \lambda_{j}\left(\Pi_{i j}+T \Phi_{i j}\right)=\Gamma_{1}^{\perp^{T}}\left(\sum_{i=1}^{N} \lambda_{i} \Psi_{1, i}\right) \Gamma_{1}^{\perp}
$$

and

$$
\sum_{i, j=1}^{N} \lambda_{i} \lambda_{j}\left(\left[\begin{array}{ccc}
\Pi_{i j}+T \Theta & T F_{1, i} & T F_{2, i} \\
* & -T M_{11} & 0 \\
* & * & -3 T M_{11}
\end{array}\right]\right)=\Gamma_{2}^{\perp^{T}}\left(\sum_{i=1}^{N} \lambda_{i} \Psi_{2, i}\right) \Gamma_{2}^{\perp}
$$

This means that the feasibility of Eq.(48c) implies the feasibility of Eq.(24c). Also the feasibility of Eq.(48d) implies the feasibility of Eq.(24d). This completes the proof.

\section{Remark 4}

The feasibility of criterion (48) implies the feasibility of the stability conditions (24) where the matrices $P, X, M_{11}, M_{12}, M_{22}$ need to be found for all subsystems. Considering a parameter dependent functional $P(\lambda(t))=\sum_{i=1}^{N} \lambda_{i}(t) P_{i}$ implies that the LMI (24) is not affine in $\lambda_{i}(t)$ and thus the convexity is lost. Therefore, we cannot simply check the conditions at the vertices of the double-layer polytope. Decoupling between LKF matrices $P, X, M_{11}, M_{12}, M_{22}$ and the data matrices $A_{i}+\Delta_{A}(t), B_{i}+\Delta_{B}(t)$, by means of introducing slack variables, allows the LMI condition (24) to be turned into a finite set of LMIs and then robust stability with less conservative conditions is achieved. This is a remarkable advantage of the proposed approach compared to the existing state of the art approaches. Furthermore, in the stability analysis, the criterion (24) has an important feature since it leads to a fairly low computational complexity due to the absence of slack variables and the small number of decision matrices.

\section{Remark 5}

It is well-known that, an LMI cab be effectively applied for a stabilization problem if there is only one coupling between a decision matrix and the system variables. The LMI stability condition (24) is not very suited for stabilization purposes due to the product terms $P\left(A_{i}+\Delta_{A}(t)\right)$, $\left(A_{i}+\Delta_{A}(t)^{T}\right) M_{11}\left(A_{i}+\Delta_{A}(t)\right)$ and $X\left(A_{i}+\Delta_{A}(t)\right)$. The stability analysis criterion (48) can be efficiently solved for the stabilization problem, because by assuming all slack variables to be equal, then there is only one coupling between a decision matrix and the system variables. 


\section{CONTROLLER SYNTHESIS}

In this section, we address synthesis of sampled-data PLPV controller for system in Eq.(18) using the results in Theorem 2. In order to improve the transient behaviour, a constrained pole placement is considered leading to robust controller design. Considering the performance criteria in the controller design procedure is an important feature of the proposed method. Consequently, the main objective is to determine the feedback gain matrices $K_{j}$ such that not only the inequality (48) holds but also additional constraints on the location of the closed-loop poles are satisfied. To this aim, we extended the $D$-stability problem (introduced in [37]) for design of robust PLPV control with pole placement constraints.

\section{Lemma 6 ([37])}

The system matrix $A$ is $D$-stable (i.e., all the poles of $A$ lie in D) if and only if there exists a symmetric matrix $Z$ such that $D(A, Z)<0, Z>0$, where $D(A, Z)$ is related with the following characteristic function $f_{D}(z)$ on the basis of the substitution $\left(Z, A Z, Z A^{T}\right)<=>(1, z, \bar{z})$ :

(i) left half plane such that $R(z)<-\alpha$

$$
D=\left\{z \in C \mid f_{D}(z)=z+\bar{z}+2 \alpha<0\right\}
$$

(ii) disk with center at $(q, 0)$ and radius $r$ :

$$
D=\left\{z \in C \mid f_{D}(z)=\left[\begin{array}{cc}
-r & q+z \\
q+\bar{z} & -r
\end{array}\right]<0\right\}
$$

(iii) conic sector centered at the origin and with inner angle $2 \theta$ :

$$
D=\left\{z \in C \mid f_{D}(z)=\left[\begin{array}{cc}
\sin \theta(z+\bar{z}) & \cos \theta(z-\bar{z}) \\
\cos \theta(z-\bar{z}) & \sin \theta(z+\bar{z})
\end{array}\right]<0\right\}
$$

The following theorem presents a set of LMIs for synthesis of robust pole placemen PLPV controller for PLPV system in (18) without considering aperiodic sampling from the states.

Theorem 3

The PLVP system in (18) is $D$-stable if there exist a matrices $M_{i j} \in R^{n}, L_{i j} \in R^{2 n}, V_{i j} \in R^{2 n}$ with $M_{i j}=M_{j i}{ }^{T}, L_{i j}=L_{j i}{ }^{T}, V_{i j}=V_{j i}{ }^{T}$, a matrix $Z \in S^{n}$ and matrices $Y_{i} \in R^{n \times n_{u}}$ such that the following LMIs feasible for $i, j=1, \ldots, N$

$$
\begin{aligned}
& H e\left[\Omega_{i j}+\Omega_{j i}+2 \alpha Z\right]<M_{i j}+M_{j i} \\
& {\left[\begin{array}{cc}
-2 r Z & 2 q Z+\Omega_{i j}+\Omega_{j i} \\
* & -2 r Z
\end{array}\right]<L_{i j}+L_{j i}} \\
& {\left[\begin{array}{cc}
\sin \theta H e\left(\Omega_{i j}+\Omega_{j i}\right) & \cos \theta\left(\Omega_{i j}-\Omega_{i j}^{T}+\Omega_{j i}-\Omega_{j i}^{T}\right) \\
* & \sin \theta H e\left(\Omega_{i j}+\Omega_{j i}\right)
\end{array}\right]<V_{i j}+V_{j i}} \\
& {\left[\begin{array}{ccc}
M_{11} & \cdots & M_{1 N} \\
\vdots & \ddots & \vdots \\
M_{N 1} & \cdots & M_{N N}
\end{array}\right]<0, \quad\left[\begin{array}{ccc}
L_{11} & \cdots & L_{1 N} \\
\vdots & \ddots & \vdots \\
L_{N 1} & \cdots & L_{N N}
\end{array}\right]<0,\left[\begin{array}{ccc}
V_{11} & \cdots & V_{1 N} \\
\vdots & \ddots & \vdots \\
V_{N 1} & \cdots & V_{N N}
\end{array}\right]<0}
\end{aligned}
$$

where $\Omega_{i j}=\left(A_{i}+\Delta_{A}(t)\right) Z+\left(B_{i}+\Delta_{B}(t)\right) Y_{j}$, then the control gains $K_{j}$ can be reconstructed by $K_{j}=Y_{j} Z^{-1}$.

Proof

Based on Lemma 6, the pole placement constraints are satisfied if there exists $Z>0$ such that

$$
D\left(\sum_{i=1}^{N} \lambda_{i}\left(A_{i}+\Delta_{A}(t)\right)+\sum_{i, j=1}^{N} \lambda_{i} \lambda_{j}\left(B_{i}+\Delta_{B}(t)\right) K_{j}, Z\right)<0
$$


Therefore, the $D$-stability condition of (18) are given by

$$
\begin{gathered}
H e[\Omega(\lambda)+\alpha Z]<0 \\
{\left[\begin{array}{cc}
-r Z & q * Z+\Omega(\lambda) \\
* & -r Z
\end{array}\right]<0} \\
{\left[\begin{array}{cc}
\sin \theta H e(\Omega(\lambda)) & \cos \theta\left(\Omega(\lambda)-\Omega^{T}(\lambda)\right) \\
* & \sin \theta H e(\Omega(\lambda))
\end{array}\right]<0}
\end{gathered}
$$

where

$$
\begin{aligned}
& \Omega(\lambda)=\sum_{i=1}^{N} \lambda_{i}\left(A_{i}+\Delta_{A}(t)\right)+\sum_{i=1}^{N} \lambda_{i}\left(B_{i}+\Delta_{B}(t)\right) \sum_{j=1}^{N} \lambda_{j} Y_{j} \\
& \sum_{j=1}^{N} \lambda_{j} Y_{j}=\sum_{j=1}^{N} \lambda_{j} K_{j} Z
\end{aligned}
$$

Hence $D$-stability condition given in (63)-(65) can be assured by (61).

In the following, an efficient procedure for robust sampled-data PLPV controller synthesis for PLPV system (18) is proposed that meets the design specifications in the sampling interval while the aperiodic sampling period is considered as a parameter. By employing the presented results in Theorem 2 the exponential stability of the designed sampled-data controller is guaranteed.

\section{Theorem 4}

Assume there exist $\bar{P}_{i} \in S_{+}^{n}, \bar{X}_{i}, \bar{M}_{11, i}, \bar{M}_{22, i} \in S^{n}, \bar{Y}_{i j} \in R^{4 n}, \bar{Z}_{i j} \in R^{6 n}$ with $\bar{Y}_{i j}=\bar{Y}_{j i}^{T}, \bar{Z}_{i j}=$ $\bar{Z}_{j i}^{T}$ and matrices $N, \bar{X}_{1, i}, \bar{M}_{12, i}, \bar{F}_{11, i}, \bar{F}_{12, i}, \bar{F}_{13, i}, \bar{F}_{21, i}, \bar{F}_{22, i}, \bar{F}_{23, i} \in R^{n \times n}, Y_{i} \in R^{n \times n_{u}}$ with positive scalars $\varepsilon_{1}, \varepsilon_{2}, \varepsilon_{3}$ such that the following LMIs feasible for $i, j=1, \ldots, N$

$$
\begin{aligned}
& {\left[\begin{array}{cc}
\bar{M}_{11, i} & \bar{M}_{12, i} \\
* & \bar{M}_{22, i}
\end{array}\right]>0} \\
& {\left[\begin{array}{cc}
I & 0
\end{array}\right]^{T} \bar{P}_{i}\left[\begin{array}{ll}
I & 0
\end{array}\right]+T\left[\begin{array}{cc}
\bar{X}_{i} & -\bar{X}_{i}+\bar{X}_{1, i} \\
* & \bar{X}_{i}-\bar{X}_{1, i}-\bar{X}_{1, i}^{T}
\end{array}\right]>0} \\
& \overline{\mathrm{E}}_{i j}+\overline{\mathrm{E}}_{j i}<\bar{Y}_{i j}+\bar{Y}_{j i} \\
& \bar{\Xi}_{i j}+\bar{\Xi}_{j i}<\bar{Z}_{i j}+\bar{Z}_{j i} \\
& {\left[\begin{array}{ccc}
\bar{Y}_{11} & \cdots & \bar{Y}_{1 N} \\
\vdots & \ddots & \vdots \\
\bar{Y}_{N 1} & \cdots & \bar{Y}_{N N}
\end{array}\right]<0,\left[\begin{array}{ccc}
\bar{Z}_{11} & \cdots & \bar{Z}_{1 N} \\
\vdots & \ddots & \vdots \\
\bar{Z}_{N 1} & \cdots & \bar{Z}_{N N}
\end{array}\right]<0}
\end{aligned}
$$

Where

$$
\overline{\mathrm{E}}_{i j}=\left[\begin{array}{cccc}
T \bar{M}_{11, i}-H e(N) & \bar{\Lambda}_{7, i} & \bar{\Lambda}_{9, i}+\left(B_{i}+\Delta_{B}(t)\right) Y_{j} & -T \bar{M}_{12, i}+N \\
* & \bar{\Lambda}_{8, i} & \bar{\Lambda}_{2, i}+\varepsilon_{1}\left(B_{i}+\Delta_{B}(t)\right) Y_{j} & -\bar{P}_{i}-T \bar{X}_{i}+\bar{\Lambda}_{3, i}+\varepsilon_{1} N \\
* & * & T \bar{M}_{22, i}+\bar{\Lambda}_{4, i} & \bar{\Lambda}_{9, i}+\bar{\Lambda}_{5, i} \\
* & * & * & T \bar{M}_{11, i}+\bar{\Lambda}_{6, i}
\end{array}\right]
$$




$$
\begin{aligned}
& \bar{\Xi}_{i j}=\left[\begin{array}{cccccc}
-\varepsilon_{2} H e(N) & \bar{\Lambda}_{10, i} & \varepsilon_{2}\left(B_{i}+\Delta_{B}(t)\right) Y_{j} & \varepsilon_{2} N & 0 & 0 \\
* & \bar{\Lambda}_{11, i} & \bar{\Lambda}_{2, i}+\varepsilon_{3}\left(B_{i}+\Delta_{B}(t)\right) Y_{j} & -\bar{P}_{i}+\bar{\Lambda}_{3, i} & T \bar{F}_{11, i} & 3 T \bar{F}_{21, i} \\
* & * & T \bar{M}_{22, i}+\bar{\Lambda}_{4, i} & \bar{\Lambda}_{5, i} & T \bar{F}_{12, i} & 3 T \bar{F}_{22, i} \\
* & * & * & \bar{\Lambda}_{6, i} & T \bar{F}_{13, i} & 3 T \bar{F}_{23, i} \\
* & * & * & * & -T \bar{M}_{11, i} & 0 \\
* & * & * & * & * & -3 T \bar{M}_{11, i}
\end{array}\right] \\
& \bar{\Lambda}_{1, i}=-\bar{X}_{i}+H e\left(-\bar{F}_{11, i}-3 \bar{F}_{21, i}\right) \\
& \bar{\Lambda}_{2, i}=-\bar{X}_{1, i}+\bar{X}_{i}+\bar{F}_{11, i}-\bar{F}_{12, i}^{T}-3 \bar{F}_{21, i}-3 \bar{F}_{22, i}^{T}-\bar{M}_{12, i} \\
& \bar{\Lambda}_{3, i}=-\bar{F}_{13, i}^{T}+6 \bar{F}_{21, i}-3 \bar{F}_{23, i}^{T}, \quad \bar{\Lambda}_{4, i}=-\bar{X}_{i}+\operatorname{He}\left(\bar{X}_{1, i}+\bar{F}_{12, i}-3 \bar{F}_{22, i}^{T}+\bar{M}_{12, i}\right) \\
& \bar{\Lambda}_{5, i}=\bar{F}_{13, i}^{T}+6 \bar{F}_{22, i}-3 \bar{F}_{23, i}^{T}, \bar{\Lambda}_{6, i}=6 \bar{F}_{23, i}+6 \bar{F}_{23, i}^{T} \\
& \bar{\Lambda}_{7, i}=\bar{P}_{i}+T \bar{X}_{i}+\left(A_{i}+\Delta_{A}(t)\right) N, \bar{\Lambda}_{8, i}=\bar{\Lambda}_{1, i}+H e\left(\varepsilon_{1}\left(A_{i}+\Delta_{A}(t)\right) N\right) \\
& \bar{\Lambda}_{9, i}=T\left(\bar{X}_{1, i}-\bar{X}_{i}+\bar{M}_{12, i}\right), \bar{\Lambda}_{10, i}=\bar{P}_{i}+\varepsilon_{2}\left(A_{i}+\Delta_{A}(t)\right) N \\
& \bar{\Lambda}_{11, i}=\bar{\Lambda}_{1, i}+\operatorname{He}\left(\varepsilon_{3}\left(A_{i}+\Delta_{A}(t)\right) N\right)
\end{aligned}
$$

Then, the sampled-data PLPV state feedback controller in Eq.(7) with vertex gains calculated as $K_{j}=Y_{j} * N^{-1}$, exponentially stabilize the PLPV system in Eq.(18) with aperiodic sampling intervals smaller than $T>0$.

Proof

We employ the stability analysis conditions presented in Eq.(48) to develop conditions for sampleddata PLPV controller design. Applying the congruent transformation $\operatorname{diag}(N, N)$ on Eqs.(48a) and (48b) with considering

$$
\begin{aligned}
& \bar{M}_{11, i}=N^{T} M_{11, i} N, \bar{M}_{12, i}=N^{T} M_{12, i} N, \bar{M}_{22, i}=N^{T} M_{22, i} N \\
& \bar{P}_{i}=N^{T} P_{i} N, \quad \bar{X}_{i}=N^{T} X_{i} N, \bar{X}_{1, i}=N^{T} X_{1, i} N, \quad N_{1}^{-1}=N
\end{aligned}
$$

the LMI conditions in Eq.(66a) and Eq.(66b) obtained.

Also LMI conditions in Eq.(66c) and Eq.(66d) with applying the congruent transformation $T_{1}=\operatorname{diag}(N, N, N, N)$ and $T_{2}=\operatorname{diag}(N, N, N, N, N, N)$ on Eq.(51) and Eq.(53) respectively and considering

$$
\begin{aligned}
& N_{2}=\varepsilon_{1} N_{1}, N_{3}=\varepsilon_{2} N_{1}, N_{4}=\varepsilon_{3} N_{1}, Y_{j}=K_{j} N \\
& \bar{F}_{11, i}=N^{T} F_{11, i} N, \bar{F}_{12, i}=N^{T} F_{12, i} N, \bar{F}_{13, i}=N^{T} F_{13, i} N \\
& \bar{F}_{21, i}=N^{T} F_{21, i} N, \bar{F}_{22, i}=N^{T} F_{22, i} N, \bar{F}_{21, i}=N^{T} F_{22, i} N \\
& \bar{Y}_{i j}=T_{1}^{T} Y_{i j} T_{1}, \bar{Z}_{i j}=T_{2}^{T} Z_{i j} T_{2} .
\end{aligned}
$$

obtain. With this concludes the proof.

\section{Remark 6}

We could combine the conditions of the sampled-data PLPV controller design in Theorem 4 and the robust PLPV controller design with constrained pole placement method in Theorem 3. By doing this, we obtained an LMI-based stabilization condition capable of achieving the pole-placement objectives and considering sample and hold constraints (aperiodic sampling period). Consequently, the proposed method can be seen as a new approach to design sampled-data PLPV controller with improved closed-loop transient behaviour.

\section{NUMERICAL EXAMPLES}

In this section, the efficiency of the proposed method is studied by three examples. In the first example, we show improvement in the performance of sampling interval bound by the proposed approach for LTI system, compared with some recent state of the art input delay approaches. The second example investigates the validity of the results of theorems stated in this paper for stability 
analysis of sampled-data LPV systems. In this example, we consider a simple second order affine LPV system with a given affine LPV controller and the MASP is computed for different rates of parameter variation. In the third example, the main application of the proposed method for sampleddata PLVP systems, has been investigated. A real world problem of balancing an inverted pendulum on a car is considered to illustrate the effectiveness of the proposed stability and design algorithm in reducing conservatism of stability analysis and improving transient behaviour of sampled-data PLPV systems.

Example 1: Consider the following well-known delay dependent stable linear system [38]:

$$
\dot{x}(t)=\left[\begin{array}{cc}
-2 & 0 \\
0 & -0.9
\end{array}\right] x(t)+\left[\begin{array}{cc}
-1 & 0 \\
-1 & -1
\end{array}\right] x\left(t_{n}\right)
$$

We use different methods to calculate the upper bound of the aperiodic sampling period that guarantees the stability of this system. The results are presented in Table I. The MASP is usually used in the literature as criterion for comparing the conservativeness of stability theorems. The bounds of the MASP are computed using the eigenvalue analysis of the corresponding transition matrix. Number of the scalar decision variables calculated based on Table II. The calculated values indicate that the proposed criteria in Theorem 1 leads to a less conservative result. Also one obtains that the results of Theorem 2 and Theorem 1 are same while Theorem 2 has higher computational complexity.

Table I. Maximum bound on the aperiodic sampling period (Example 1).

\begin{tabular}{lcccccc}
\hline Method & Th. bounds & {$[12]$} & {$[11]$} & {$[33]($ Theorem 2) } & Theorem 1 & Theorem 2 \\
\hline$T_{m}$ & 3.272 & 1.563 & 2.515 & 2.515 & 2.627 & 2.627 \\
\hline No. of variables & - & 12 & 34 & 42 & 36 & 52 \\
\hline
\end{tabular}

Table II. Numerical complexity of the different methods.

\begin{tabular}{|l|c|c|c|}
\hline Method & No. of decision variables & No. of LMIs & Maximum order of LMI \\
\hline$[12]$ & $2 n^{2}+2 n$ & 5 & $4 n$ \\
\hline$[11]$ & $8 n^{2}+n$ & 3 & $4 n$ \\
\hline$[33]$ & $10 n^{2}+n$ & 4 & $4 n$ \\
\hline Theoream 1 & $8 n^{2}+2 n$ & 5 & $4 n$ \\
\hline Theoream 2 & $12 n^{2}+2 n$ & 5 & $5 n$ \\
\hline
\end{tabular}

Example 2: Consider the following LPV system.

$$
\dot{x}(t)=\left[\begin{array}{cc}
0 & 1 \\
0.1 & 0.4+0.6 \sigma(t)
\end{array}\right] x(t)+\left[\begin{array}{l}
0 \\
1
\end{array}\right] u(t)
$$

with $|\sigma(t)| \leq 1$ and $|\dot{\sigma}(t)| \leq 0.2$. In [27], the following stabilizing sampled-data controller designed for a given maximum bound on aperiodic sampling period $\left(T_{m}=400 \mathrm{~ms}\right)$.

$$
k=\left[\begin{array}{ll}
-1.21 & -2.35
\end{array}\right]+\left[\begin{array}{ll}
0.149 & -0.15
\end{array}\right] \sigma\left(t_{n}\right)
$$

We can represent (70) accurately in PLVP form. According to (13), we have

$$
\left[\begin{array}{c}
\dot{\alpha}_{1}(t) \\
\dot{\alpha}_{1}(t)
\end{array}\right]=\left[\begin{array}{cc}
-0.5 & 0.5 \\
0.5 & 0.5
\end{array}\right]\left(\left[\begin{array}{cc}
-0.2 & 0.2 \\
1 & 1
\end{array}\right]\left[\begin{array}{l}
\beta_{1}(t) \\
\beta_{2}(t)
\end{array}\right]-\left[\begin{array}{l}
0 \\
1
\end{array}\right]\right) .
$$

Therefore, $\dot{\alpha}_{i}(t) \in[-0.1,0.1]$ for $i=1,2$.

In the following, we evaluate conservativeness of the stability conditions for different rates of parameter variation. Table III shows maximum bound on the sampling period for different existing approach for stability analysis of sampled-data LPV systems. It is verified that the proposed 
Table III. Maximum bound on the aperiodic sampling period of sampled-data LPV system

\begin{tabular}{|l|c|c|c|}
\hline Th. bounds on $\dot{\sigma}(t)$ & 0.2 & 0.6 & 1 \\
\hline$[22]$ & 0.39 & 0.38 & 0.36 \\
\hline$[27](P)$ & 0.65 & 0.58 & 0.52 \\
\hline$[27](P(\sigma(t)))$ & 0.73 & 0.69 & 0.66 \\
\hline Theoream 1 & 0.71 & 0.66 & 0.65 \\
\hline Theoream 2 & 0.77 & 0.73 & 0.69 \\
\hline
\end{tabular}

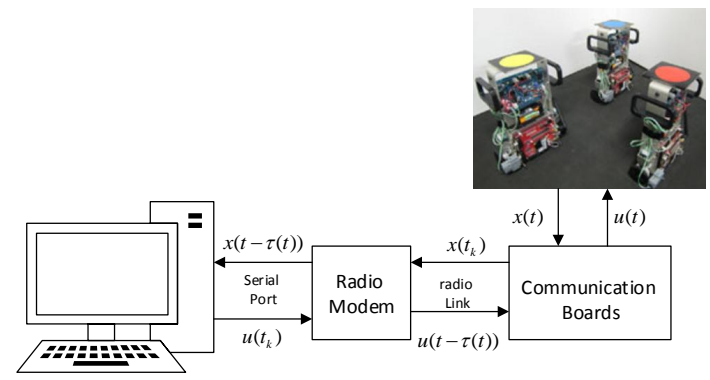

Figure 3. Control architecture of inverted pendulum on a car.

approach in Theorem 2 has higher performance than the loop functional [27] and a smooth LKF [22] approaches and reducing conservatism of stability.

Example 3: Consider the following equations of motion for the pendulum on a car

$$
\begin{aligned}
& \dot{x}_{1}=x_{2} \\
& \dot{x}_{2}=\frac{g \sin (x 1)-a m l x_{2}^{2} \sin \left(2 x_{1}\right) / 2-a \cos \left(x_{1}\right) u}{4 l / 3-a m l \cos ^{2}\left(x_{1}\right)} .
\end{aligned}
$$

where $x_{1}$ denotes the angle of the pendulum from the vertical, $x_{2}$ is the angular velocity, $g=$ $9.8 \mathrm{~m} / \mathrm{s}^{2}$ is the gravity, $m$ is the mass of the pendulum, $M$ is the mass of the cart, $2 l$ is the length of the pendulum, and $u$ is the force applied to the cart, $a=1 /(m+M), m=2 \mathrm{~kg}, M=8 \mathrm{~kg}, 2 \mathrm{l}=1 \mathrm{~m}$ are chosen for simulation. The following polytopic quasi-LPV model of the inverted pendulum on a car systems presented in [39].

$$
\begin{aligned}
& A_{1}=\left[\begin{array}{cc}
0 & 1 \\
17.29 & 0
\end{array}\right], \quad B_{1}=\left[\begin{array}{c}
0 \\
-0.176
\end{array}\right] \\
& A_{2}=\left[\begin{array}{cc}
0 & 1 \\
12.63 & 0
\end{array}\right], \quad B_{2}=\left[\begin{array}{c}
0 \\
-0.077
\end{array}\right] \\
& \alpha_{1}(t)=\left(1-\frac{1}{1+\exp \left(-7\left[x_{1}-\pi / 4\right]\right)}\right)\left(\frac{1}{1+\exp \left(-7\left[x_{1}+\pi / 4\right]\right.}\right) \\
& \alpha_{2}(t)=1-\alpha_{1}(t)
\end{aligned}
$$

The sate of the inverted pendulum on a car are measured at sampling instants, $t_{n}$ and through a radio modem sent to the PC. The PC computes desirable control input and sent it to inverted pendulum on a car, see Fig.(3).

We design the following controller 1 with pole placement in regions $R(z) \leq-3$, and controller 2 without the pole placement constraints for $T=0.15$ and $\left|\dot{\alpha}_{i}\right| \leq 0.1$.

$$
\begin{aligned}
& \text { controller } 1=\left[\begin{array}{ll}
191.31 & 53.11
\end{array}\right] \alpha_{1}\left(t_{n}\right)+\left[\begin{array}{ll}
339.98 & 101.23
\end{array}\right] \alpha_{2}\left(t_{n}\right) \\
& \text { controller } 2=\left[\begin{array}{ll}
250.16 & 63.23
\end{array}\right] \alpha_{1}\left(t_{n}\right)+\left[\begin{array}{lll}
449.61 & 122.84
\end{array}\right] \alpha_{2}\left(t_{n}\right)
\end{aligned}
$$


We also considered the following $H_{\infty}$ controller designed in [40].

$$
K_{1}=\left[\begin{array}{ll}
409.75 & 99.23
\end{array}\right], K_{2}=\left[\begin{array}{ll}
512.91 & 121.79
\end{array}\right] \text {. }
$$

Fig. 4 and Fig. 5 show comparison of the response of closed loop of system for an initial condition $x_{1}(0)=[1.2-1]$. Fig .6 shows applied control signals. We observe that designed controller 1 has the suitable performance despite the variable sampling. The figures clearly demonstrate that in the presence of aperiodic sampling period and despite of the uncertainties on scheduling parameters, the states tend to the origin with an acceptable settling time. Also the control signals are enough smooth and variation of sampling is not effective.

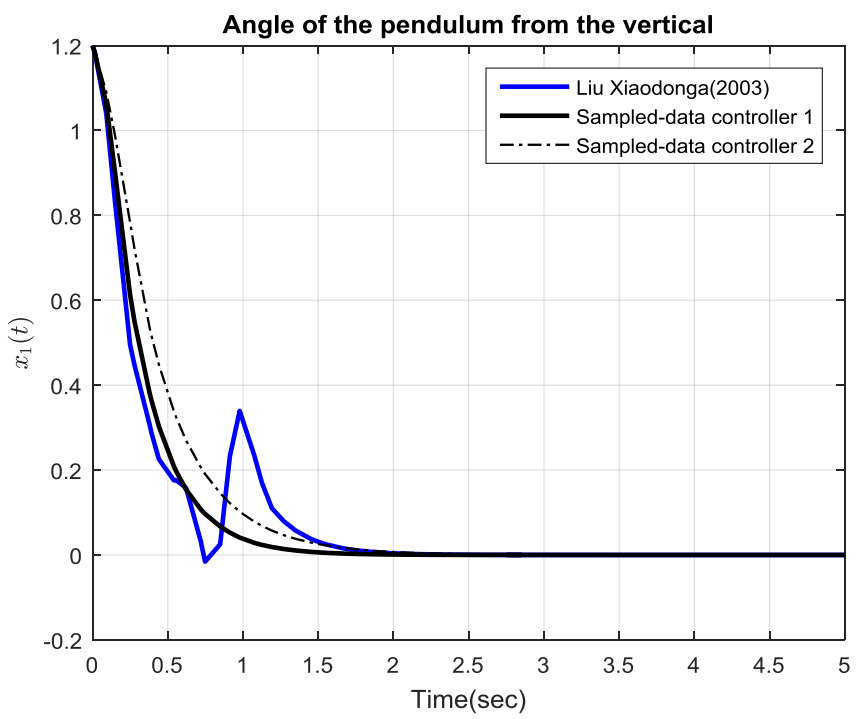

Figure 4. Comparison of the response of system under the controller obtained by Theorem 4 .

The criteria proposed in Theorem 2 is applied to identify the upper bounded of aperiodic sampling period of the PLPV model of inverted pendulum on a car with different rate of parameter variation for three different controllers. Table IV shows the results. This comparison shows the advantageous of the proposed sampled-data PLPV control design scheme for networked control systems.

Table IV. Maximum bound on the aperiodic sampling period of sampled-data LPV system

\begin{tabular}{|l|c|c|c|}
\hline Th. bounds on $\dot{\alpha}_{i}(t)$ & 0.1 & 0.3 & 1 \\
\hline controller 1 & 0.168 & 0.161 & 0.147 \\
\hline controller 2 & 0.202 & 0.194 & 0.175 \\
\hline Liu Xiaodonga [40] & 0.108 & 0.105 & 0.101 \\
\hline
\end{tabular}

\section{CONCLUSION}

In this paper we proposed a new methodology for exponential stability analysis and design of the closed loop sampled-data PLPV systems with aperiodic sampling. The proposed method has three important features: (i) new form of discrete LKF with the Wirtingers inequality was proposed that provides less conservative stability analysis condition of sampled-data PLPV systems rather than existing methods, (ii) double-layer description utilized to model inequality among real and sampled parameters which reduced the complexity and in combination with the proposed stability 


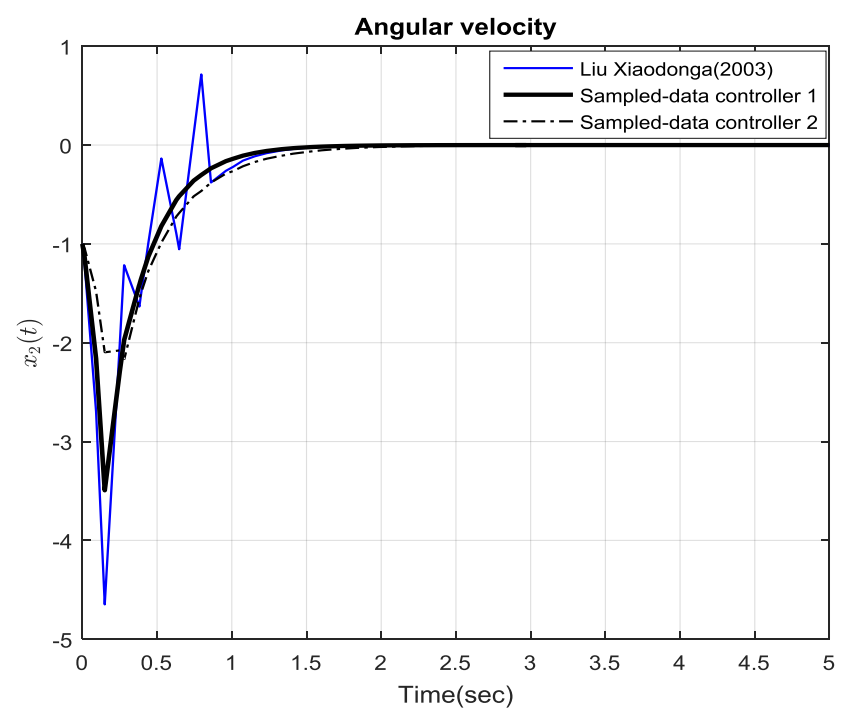

Figure 5. Comparison of the response of system under the controller obtained by Theorem 4 .

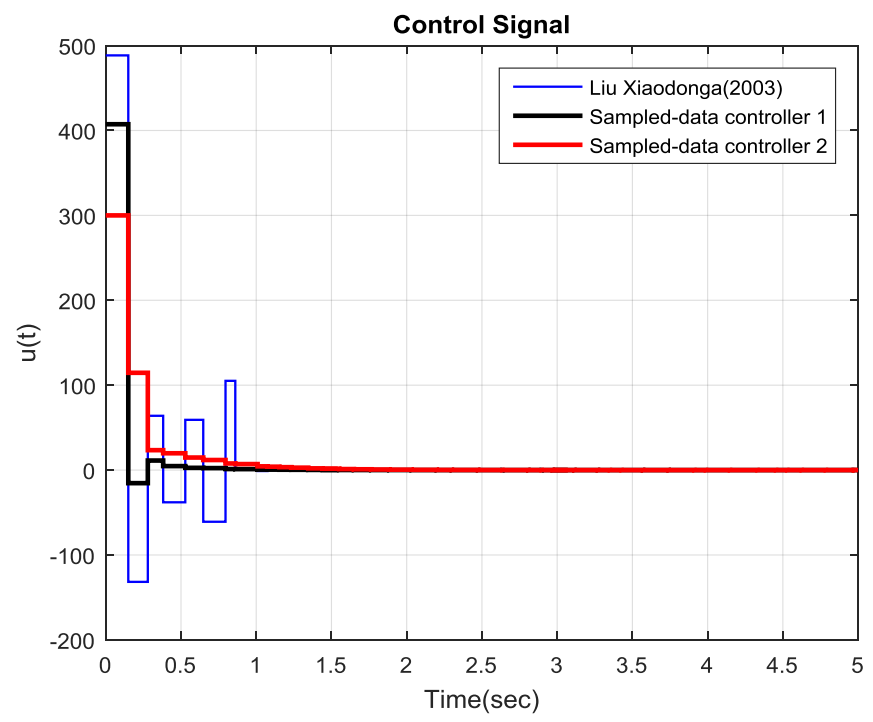

Figure 6. Control signal with $T=0.15$.

conditions has suitable performance in MASP computations, and (iii) a sampled-data polytopic LPV controller was synthesized regarding the aperiodic sampling and the proposed pole placement technique provided a viable control strategy. The effectiveness of the approach was verified by means of numerical simulations that illustrated a significant robustness for aperiodic sampling without performance degrading. The computational complexity of the proposed approach for large systems with large numbers of scheduling parameters is an important issue need to be considered. Finally, it is noted that the complexity reduction of the proposed method and extending it to the output feedback control problems is our current ongoing work. To this aim, the idea is to use less conservative available tools rather than the Wirtinger-based inequalities (see e.g. [41, 42, 43]) to provide a smaller bounding gap. 


\section{REFERENCES}

1. Hespanha JP, Naghshtabrizi P, Xu Y. A survey of recent results in networked control systems. Proc-IEEE 2007; 95(1):138.

2. Yang F, Zhang H, Liu Z, Li R. Delay-dependent resilient-robust stabilisation of uncertain networked control systems with variable sampling intervals. International Journal of Systems Science 2014; 45(3):497-508.

3. Orihuela L, Millán P, Vivas C, Rubio FR. Event-based $H_{2} / H_{\infty}$ controllers for networked control systems. International Journal of Control 2014; 87(12):2488-2498.

4. Park MJ, Kwon OM, Seuret A. Weighted consensus protocols design based on network centrality for multi-agent systems with sampled-data. IEEE Transactions on Automatic Control 2017; 62(6):2916-2922.

5. Yamamoto Y. A function space approach to sampled data control systems and tracking problems. IEEE Transactions on Automatic Control 1994; 39(4):703-713.

6. Mirkin L, et al.. Some remarks on the use of time-varying delay to model sample-and-hold circuits. IEEE Transactions on Automatic Control 2007; 52(6):1109.

7. Naghshtabrizi P, Hespanha JP, Teel AR. Exponential stability of impulsive systems with application to uncertain sampled-data systems. Systems \& Control Letters 2008; 57(5):378-385.

8. van de Wouw N, Naghshtabrizi P, Cloosterman M, Hespanha JP. Tracking control for sampled-data systems with uncertain time-varying sampling intervals and delays. International Journal of Robust and Nonlinear Control 2010; 20(4):387-411.

9. Seuret A. A novel stability analysis of linear systems under asynchronous samplings. Automatica 2012; 48(1):177182.

10. Seuret A, Briat C. Stability analysis of uncertain sampled-data systems with incremental delay using loopedfunctionals. Automatica 2015; 55:274-278.

11. Fridman E. A refined input delay approach to sampled-data control. Automatica 2010; 46(2):421-427.

12. Liu K, Fridman E. Wirtingers inequality and Lyapunov-based sampled-data stabilization. Automatica 2012; 48(1):102-108.

13. Mazenc F, Malisoff M, Dinh TN. Robustness of nonlinear systems with respect to delay and sampling of the controls. Automatica 2013; 49(6):1925-1931.

14. Karafyllis I, Kravaris C. Global stability results for systems under sampled-data control. International Journal of Robust and Nonlinear Control 2009; 19(10):1105-1128.

15. Nesic D, Teel AR, Carnevale D. Explicit computation of the sampling period in emulation of controllers for nonlinear sampled-data systems. IEEE Transactions on Automatic Control 2009; 54(3):619-624.

16. Lee S, Park M, Kwon O, Sakthivel R. Synchronization of lur'e systems via stochastic reliable sampled-data controller. Journal of the Franklin Institute 2017; 354(5):2437-2460.

17. Lee S, Park M, Kwon O, Sakthivel R. Advanced sampled-data synchronization control for complex dynamical networks with coupling time-varying delays. Information Sciences 2017;

18. Hoffmann C, Werner H. A survey of linear parameter-varying control applications validated by experiments or high-fidelity simulations. IEEE Transactions on Control Systems Technology 2015; 23(2):416-433.

19. Henry D, Cieslak J, Zolghadri A, Efimov D. $H_{\infty}$ LPV solutions for fault detection of aircraft actuator faults: Bridging the gap between theory and practice. International Journal of Robust and Nonlinear Control 2015; 25(5):649-672.

20. Tan K, Grigoriadis KM. State-feedback control of LPV sampled-data systems. Mathematical Problems in Engineering 2000; 6(2-3):145-170.

21. Tan K, Grigoriadis KM, Wu F. Output-feedback control of LPV sampled-data systems. International Journal of Control 2002; 75(4):252-264.

22. Ramezanifar A, Mohammadpour J, Grigoriadis K. Sampled-data control of LPV systems using input delay approach. IEEE 51st Annual Conference on Decision and Control (CDC), IEEE, 2012; 6303-6308.

23. Ramezanifar A, Mohammadpour J, Grigoriadis KM. Sampled-data control of linear parameter varying time-delay systems using state feedback. American Control Conference (ACC), IEEE, 2013; 6847-6852.

24. Ramezanifar A, Mohammadpour J, Grigoriadis KM. Output-feedback sampled-data control design for linear parameter-varying systems with delay. International Journal of Control 2014; 87(12):2431-2445.

25. Shi T, Su H. Sampled-data mpc for LPV systems with input saturation. IET Control Theory \& Applications 2014; 8(17):1781-1788

26. Lee S, Kwon O. Quantised mpc for LPV systems by using new lyapunov-krasovskii functional. IET Control Theory \& Applications 2016; 11(3):439-445.

27. Da Silva JG, Moraes V, Flores J, Palmeira A. Sampled-data LPV control: a looped functional approach. IFACPapersOnLine 2015; 48(26):19-24.

28. Takagi T, Sugeno M. Fuzzy identification of systems and its applications to modeling and control. IEEE transactions on systems, man, and cybernetics 1985; (1):116-132.

29. Boustany N. Unified algebraic approach to linear control design. re skelton et al. APPLIED MECHANICS REVIEWS 1998; 51:B89-B89.

30. Xie L, de Souza Carlos E. Robust h/sub infinity/control for linear systems with norm-bounded time-varying uncertainty. IEEE Transactions on Automatic Control 1992; 37(8):1188-1191.

31. Seuret A, Gouaisbaut F. Wirtinger-based integral inequality: application to time-delay systems. Automatica 2013; 49(9):2860-2866.

32. Gao H, Sun W, Shi P. Robust sampled-data $H_{\infty}$ control for vehicle active suspension systems. IEEE Transactions on Control Systems Technology 2010; 18(1):238-245.

33. Zhang CK, Jiang L, He Y, Wu H, Wu M. Stability analysis for control systems with aperiodically sampled data using an augmented lyapunov functional method. IET Control Theory \& Applications 2013; 7(9):1219-1226.

34. Xu S, Sun G, Sun W. Fuzzy logic based fault-tolerant attitude control for nonlinear flexible spacecraft with sampleddata input. Journal of the Franklin Institute 2017; 354(5):2125-2156. 
35. Xu S, Sun G, Sun W. Reliable sampled-data vibration control for uncertain flexible spacecraft with frequency range limitation. Nonlinear Dynamics 2016; 86(2):1117-1135.

36. Gahinet P, Apkarian P. A linear matrix inequality approach to $H_{\infty}$ control. International journal of robust and nonlinear control 1994; 4(4):421-448.

37. Chilali M, Gahinet P. $H_{\infty}$ design with pole placement constraints: an LMI approach. IEEE Transactions on automatic control 1996; 41(3):358-367.

38. Kao CY, Rantzer A. Stability analysis of systems with uncertain time-varying delays. Automatica 2007; 43(6):959970.

39. Cao SG, Rees NW, Feng G. Stability analysis and design for a class of continuous-time fuzzy control systems. International Journal of Control 1996; 64(6):1069-1087.

40. Xiaodong L, Qingling Z. New approaches to $H_{\infty}$ controller designs based on fuzzy observers for TS fuzzy systems via LMI. Automatica 2003; 39(9):1571-1582.

41. Zhang CK, He Y, Jiang L, Wu M, Zeng HB. Stability analysis of systems with time-varying delay via relaxed integral inequalities. Systems \& Control Letters 2016; 92:52-61.

42. Liu K, Seuret A, Xia Y. Stability analysis of systems with time-varying delays via the second-order bessel-legendre inequality. Automatica 2017; 76:138-142.

43. Zhang CK, He Y, Jiang L, Wu M, Wang QG. An extended reciprocally convex matrix inequality for stability analysis of systems with time-varying delay. Automatica 2017; 85:481-485. 


\section{University Library}

\section{- M M I N E R VA A gateway to Melbourne's research publications}

Minerva Access is the Institutional Repository of The University of Melbourne

Author/s:

Hooshmandi, K;Bayat, F;Jahed-Motlagh, MR;Jalali, AA

Title:

Stability analysis and design of nonlinear sampled-data systems under aperiodic samplings

Date:

2018-05-10

Citation:

Hooshmandi, K., Bayat, F., Jahed-Motlagh, M. R. \& Jalali, A. A. (2018). Stability analysis and design of nonlinear sampled-data systems under aperiodic samplings. International Journal of Robust and Nonlinear Control, 28 (7), pp.2679-2699. https://doi.org/10.1002/rnc.4043.

Persistent Link:

http://hdl.handle.net/11343/283554 